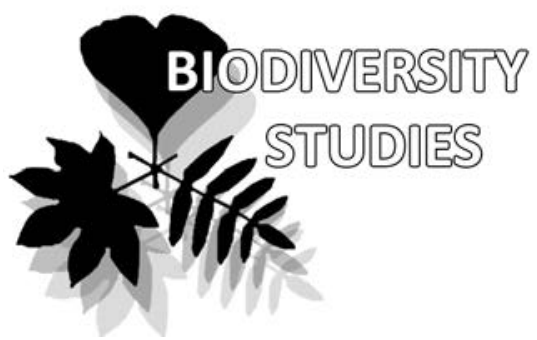

\section{The vascular flora of the Sutai Khairkhan Mountain Nature Reserve, Mongolia}

Vanjil Gundegmaa ${ }^{1}$, Tsogtbayar Dashmaa ${ }^{1}$, Chuntai Bilegtmandakh ${ }^{2}$, Zagarjav Tsegmed ${ }^{3}$, Joscelyn Norris ${ }^{4}$, Batlai Oyuntsetseg, Andrey S. Erst ${ }^{6} \&$ Shukherdorj Baasanmunkh ${ }^{7 *}$
Vanjil Gundegmaa ${ }^{1}$

e-mail: gundegmaa@msue.edu.mn

Tsogtbayar Dashmaa

e-mail:dashmaa@msue.edu.mn

Chuntai Bilegtmandakh ${ }^{2}$

e-mail: bilegtmandakh.ch@,khu.edu.mn

Zagarjav Tsegmed ${ }^{3}$

e-mail: tsegmidzagarjaw@gmail.com

Joscelyn Norris ${ }^{4}$

e-mail: joscie.norris@gmail.com

Batlai Oyuntsetseg ${ }^{5}$

e-mail: oyunaa@num.edu.mn

Andrey S. Erst

e-mail: erst_andrew@yahoo.com

Shukherdorj Baasanmunkh ${ }^{7 *}$ e-mail: baasanmunkh.sh@gmail.com

${ }^{1}$ Department of Biology, School of Mathematics and Natural Sciences, Mongolian National University of Education, Ulaanbaatar, Mongolia ${ }^{2}$ Department of Geography and Geology, Khovd University, Khovd, Mongolia

${ }^{3}$ Laboratory of Flora and Plant Systematics, Botanic Garden and Research Institute, Mongolian Academy of Sciences, Ulaanbaatar, Mongolia

${ }^{4}$ Reubenstein School of Environment and Natural Resources, University of Vermont, Vermont, United States

${ }^{5}$ Department of Biology, School of Arts and Science, National University of Mongolia, Ulaanbaatar, Mongolia

${ }^{6}$ Central Siberian Botanical Garden SB RAS, Novosibirsk, Russia

${ }^{7}$ Department of Biology and Chemistry, Changwon National University, Changwon, South Korea

* corresponding author

Manuscript received: 28.08 .2021 Review completed: 05.01.2022 Accepted for publication: 26.01.2022 Published online: 28.01.2022

\begin{abstract}
A B S T R A C T
In this study we examined the floristic diversity of the Sutai Khairkhan Mountain Nature Reserve in western Mongolia's Altai Mountain range. This nature reserve area was established in 2019 and compared to nearby reserves, its flora is relatively understudied. From field surveys in 2014, 2019, and 2020, we collected about 400 herbarium specimens from various habitats and different altitudes in the reserve. We identified total of 317 taxa including 10 subspecies and three varieties of vascular plants belonging to 157 genera and 45 families. Among these, five species are nationally endemic, 27 species are endemic to Altai Mountains, and 37 species are threatened, including two critically endangered, 11 endangered, 16 vulnerable, and eight near threatened. In addition, we rediscovered Microula tibetica var. pratensis (Maxim.) W.T. Wang after 40 years. This first complete checklist of the SKMNR flora amplifies the value of protecting the diverse and threatened plants in the reserve and creates a baseline to assess future population changes.
\end{abstract}

Keywords: Microula tibetica, vascular plants, protected area, west Mongolia, Altai endemic.

\section{P E 3 Ю M E}

Гүндэгмаа В., Аашмаа Ц., Билэгтмандах Ч., ЦэгмэА 3., Норрис Ж., Оюунцэцэг Б., Эрст А.С., Баасанмунх Ш., Фцора сосудистых растений заповедникй Гора Сутай Хайрхан, Монгомия. Изучено флористическое разнообразие горного заповедника Сутай Хайрхан (SKMNR), расположенного на территории горного хребта А^тай в Западной Монголии. Заповедник был основан в 2019 году и, по сравнению с соседними заповедниками, его флора относительно мало изучена. В ходе полевых исследований, продолжающихся с 2014 по 2020 г., было собрано около 400 гербарных образцов из разАичных местообитаний в разных высотных поясах. В результате ревизии гербарных фондов и полевых исследований было выявлено 317 таксонов, которые включают 10 подвидов и три разновиАности сосудистых растений, относящихся к 157 родам и 45 семействам. Пять виАОв яв яются энАемиками Монголии, 27 виАОв яв яются энАемиками гор ААтая и 37 виАов находятся поА угрозой исчезновения, в том числе Ава - в критическом состоянии, 11 исчезающих и 16 уязвимых и восемь находящихся поА угрозой исчезновения. Кроме того, спустя 40 мет, был заново найлен Microula tibetica var. pratensis (Maxim.) W.T. Wang. Этот виА считался исчезнувшим Аля этой территории. Изучение известных местонахождений и вновь полученные Аанные Аали возможность оценить статус редкости этого таксона на основе критериев МСОП - исчезающий вид в Монголии. Полученный список флоры SKMNR имеет важное значение Аля природоохранных целей, а также является основой Аля оценки будущих изменений в популяциях реАких и эндемичных растений заповдника.

КАючевые слова: Microula tibetica, сосудистые растения, охраняемая территория, 3ападная Монголия, эндемик А^тая

The Altai Mountains, also known as the Altai Mountain Country (AMC), are located in the western part of the Altai-Sayan Ecoregion, and lies at the junction of four countries: Russia, Kazakhstan, China, and Mongolia (Kamelin 2005, Vaganov et al. 2019). One of the biggest parts of the AMC is located in western Mongolia which includes the Khovd (Kho), Mongolian Altai (MA), and Dzungarian Gobi (DzG) phytogeographical regions according to Grubov (1982). West Mongolia is a center of high vascular plant diversity comprises approximately 2000 taxa, which contains large number of endemic and threatened plant species compared to other parts of Mongolia (Pyak et al. 2008, Olonova et al. 2010, Oyuntsetseg et al. 2017, Baasanmunkh et al. 2019a, b, 2021a). Several high elevation mountains in the Mongolian part of the Altai Mountain range include Munkhkhairkhan, Jargalant, Bumbat, Baatar, Sutai Khairkhan, and Tsambagarav. The Sutai Khairkhan Mountain Nature Reserve (SKMNR) belongs to the Mongolian Altai (MA) phytogeographical region in western Mongolia (Grubov 1982). 
In the past, the flora of western Mongolia was quite well studied by botanists mainly from Mongolia, Russia and Germany. A number of researchers have given much attention to the new species and new records of the vascular plants from western Mongolia (Zhao et al. 2018, Gundegmaa \& Munkh-Erdene 2018, Nobis et al. 2019, Pyak \& Pyak 2018, Pyak et al. 2020, Ovchinnikova 2020, Baasanmunkh et al. 2020, Shiga et al. 2020). Additionally, in western Mongolia, a few studies on floristic diversity have recently been conducted in the protected areas such as Strictly Protected Areas (SPA), National Parks (NP) as well as Nature Reserves (NR). For example, Oyuntsetseg et al. (2017) contributed a floristic survey on the Munkhkhairkhan NP and Baasanmunkh et al (2021a) provided an annotated checklist of vascular plants in the Dzungarian Gobi region including the Great Gobi B Strictly Protected Area (SPA) and part of Bulgan Ikh Ongog NP. Despite this research, several nature reserve areas, particularly in western Mongolia, such as the SKMNR, are still underexplored, particularly in regards to documenting their floristic diversity, endemism, and threatened vascular plants. The SKMNR covers a 172713.9-hectare area and was established in 2019 under the Ministry of Environment, Green Development and Tourism (https://eic.mn/spa/). Moreover, the Sutai Khairkhan Mountain is one of the sacred mountains in Mongolia, according to UNESCO (https://whc.unesco.org/en/ tentativelists/6068/).

To date, approximately 3200 species of vascular plants have been recognized in Mongolia (Urgamal et al. 2014, 2019a, Shiga et al. 2020, Baasanmunkh et al. 2021b, Yano et al. 2021). In addition, several new species and new records were described from western Mongolia (Zhao et al. 2018, Nobis et al. 2019, Pyak et al. 2019, Ovchinnikova 2020). More recently, Saussurea odorata E. Pjak was described as a new species to science from the Mongolian Altai (MA) region (Pyak et al. 2020) and critically endangered Saussurea bogedaensis Yu J.Wang \& J.Chen was also newly discovered in Mongolia in the Dzungarian Gobi region (Baasanmunkh et al. 2020). Additionally, nine aquatic plants were recently found at the Khar-Us Lake NP in western Mongolia (Shiga et al. 2020). As shown by these recent publications, a number of new plant species are still being found and many unknown species remain to be discovered in Mongolia.

Based on our three years of field observations, this paper provides the first full checklist of floristic diversity of the Sutai Khairkhan Mountain NR area and reviews the conservation status of rare species.

\section{MATERIAL AND METHODS}

The Sutai Khairkhan Mountain Nature Reserve $\left(46^{\circ} 40^{\prime}-46^{\circ} 34^{\prime} \mathrm{N} 99^{\circ} 26^{\prime}-93^{\circ} 53^{\prime} \mathrm{E}\right)$ is located in the Khovd and Gobi-Altai provinces of Mongolia and belongs to the MA phytogeographical region of Mongolia (Figs 1, 2). The dominant vegetation types here are alpine, high mountain, and mountain steppe. The Sutai Khairkhan, the mountain at the center of the SKMNR, rises $4000 \mathrm{~m}$ a.s.l. and is one of the highest mountains in the Altai Mountain system.

The field study was conducted in 2014, 2019 and 2020. During this time, we collected approximately 400 herbarium specimens from different elevations and various vegetation types. Species were identified using the standard guides of Grubov (1982), Flora of Mongolian series, Flora of China (eFlores 2008), and Endemic Plants of the Altai Mountain Country (Pyak et al. 2008). The accepted name of each species follows the Plants of the World Online (POWO 2020, http://www.plantsoftheworldonline.org/). The status of national endemic plants follows Baasanmunkh et al. (2021c), and endemic plants of the AMC follows Erst et al. (2021). Threatened plant species were based on Nyambayar et al. (2011), Oyuntsetseg et al. (2018) and Urgamal et al. (2019b). The regional distribution points for each species were based on Grubov (1982), Gubanov (1996), Urgamal et al (2014), German (2015) and Baasanmunkh et al. (2021a). The voucher specimens were deposited in the herbarium of the National University of Mongolia (UBU). The species data of each protected areas were derived from literature (if available) as well as Mongolian Protected Areas (http:/ / mpa.gov.mn/). The grid distribution map of Microula tibetica var. pratensis (Maxim.) W.T. Wang was created in ArcGis, using the same approach as Baasanmunkh et al. (2022).

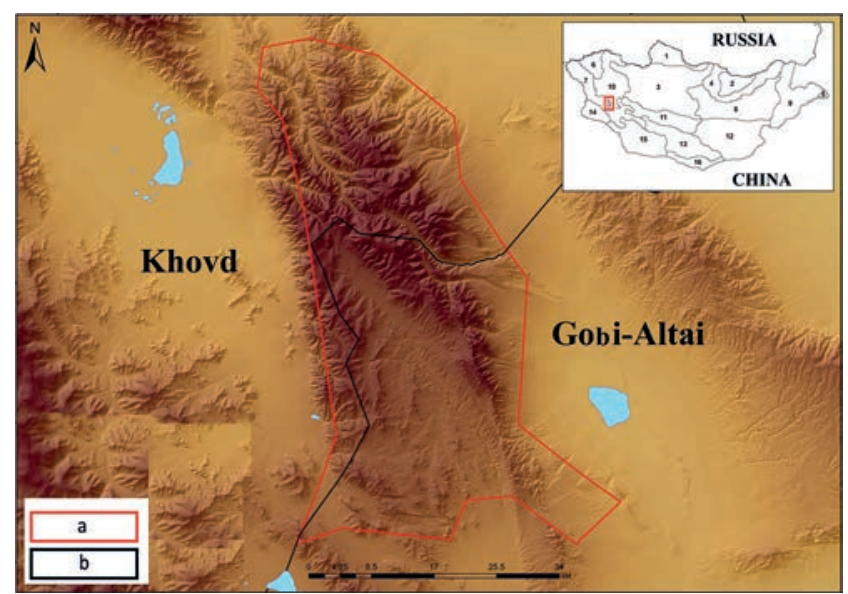

Figure 1 Study area of the Sutai Khairkhan Mountain Nature Reserve (a) in relations to a boundary between provinces (b) and floristic regions of Mongolia (1-16) after Grubov (1982)

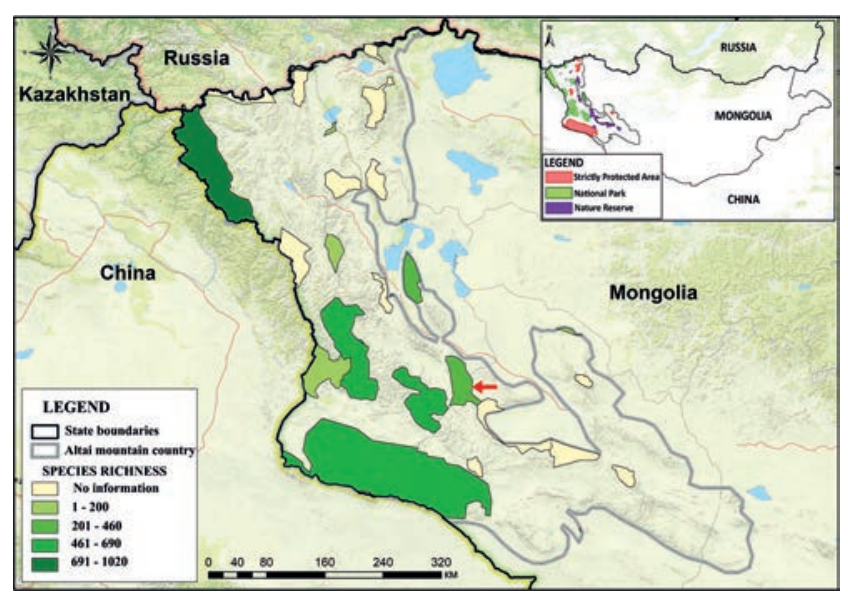

Figure 2 The species richness pattern of the protected areas in the Altai Mountain range of western Mongolia. The SKMNR is marked by a red arrow. 


\section{RESULTS}

From three years of field surveys, approximately 400 herbarium collections were gathered; many of these samples have one or several duplicates. From the collections, we identified 317 vascular plant taxa (10 subspecies and 3 varieties) belonging to 157 genera and 45 families in the SKMNR (Fig. 2; Appendix 1). The angiosperms are represented by 308 species belonging to 151 genera. Additionally, four species of fern and fern allies in three genera as well as three species of gymnosperms belonging to three genera, were noted. Among these, the family with the greatest species diversity was Asteraceae with 48 species, followed by Fabaceae (27 species), Rosaceae (27 species), Caryophyllaceae (24 species) and Poaceae (23 species). The largest genus was Potentilla L. with 17 species followed by Oxytropis DC. (13 species), Artemisia L. (11 species) and Saussurea DC. (9 species), shown in Table 1.

In addition, 11 species were newly found in the MA region (Table 2) which were previously only recorded in several other phytogeographical regions of the country. For example, Microula tibetica var. pratensis (Maxim.) W.T. Wang was found again in the MA region, after 40 years; we provided more information about this rediscovery below (see Taxonomic treatment).

We also found 37 threatened plants including critically endangered (two species), endangered (11 species), vulnerable (16 species) and near threatened (8 species) from the SKMNR (Table 3). Examples of some rare and threatened plants were also photographed for documentation, namely Leiospora exscapa (C.A. Mey.) F. Dvorák (Fig. 3A), Corydalis inconspicua Bunge ex Ledeb. (Fig. 3B), Saussurea glacialis Herder (Fig. 3C), Draba altaica (C.A. Mey.) Bunge (Fig. 3D), and Waldheimia tridactylites Kar. \& Kir. (Fig. 3E) in this area. For example, $S$. glacialis was frequently distributed in Mongolia but there are no wild photographs to date.

\section{DISCUSSION}

The Mongolian Altai region has one of the highest diversities of vascular plants compared to other regions of Mongolia (Neuffer et al. 2003, Urgamal et al. 2014, Gundegmaa et al. 2018, Baasanmunkh et al. 2019, 2021). Approximately 1700 species of vascular plants have been recognized in this region which accounts for $53 \%$ of Mongolia's vascular plants (Gubanov 1996, Neuffer et al. 2003, Urgamal et al. 2014, Bekket et al. 2015, Oyuntsetseg et al. 2017, Baasanmunkh et al. 2021). In addition, we found 11 species which had not been recorded before in the MA region, according to Urgamal et al. (2014). Furthermore, we re-discovered the wild population of Microula tibetica var. pratensis (Maxim.) W.T. Wang after 40 years.

The Altai Mountain parts of Mongolia has a large number of endemic plants and threatened plant species compared to other parts of Mongolia (Pyak et al. 2008, Oyuntsetseg et al. 2018, Baasanmunkh et al. 2019b, Erst et al. Nature Reserve.
2021). Recently, Erst et al. (in press) updated and revised the checklist of endemic plants of the AMC which contains 302 species with representative herbarium barcodes. Among these, we documented 27 species in the SKMNR which shares about $9 \%$ of the species in the AMC. Furthermore, Erst et al. (in press) determined that the species richness is 312 species based on botanical-geographical subdivisions of the AMC. According to Erst et al. (2021), there are two subdivisions, namely Khobdo-Tonkhil (ZM3) and SouthMongolia (UM), that have relatively few species with 10 and 4 species, respectively. However, our results show a much higher species richness of about 27 endemic plants that occur in the SKMNR ZM3 subdivision because of our more comprehensive field surveys.

Since 2011, over 600 species' regional conservation status in Mongolia has been assessed (Nyambayar et al. 2011, Oyuntsetseg et al. 2018, Baasanmunkh et al. 2019, Urgamal et al. 2019, Baasanmunkh et al. 2021c). We documented about 30 of these threatened species in the SKMNR.

The Sutai Khairkhan Mountain NR is a small area compared to the whole Altai Mountain range; however, it contains a high diversity of vascular plants including endemic and threatened plant species (Fig. 2). Additionally, because it is one of the largest mountain ranges in western Mongolia, it's flora must not be overlooked as it is significant to our understanding of the conservation of these

Table 1. The most represented families $(\geq 14$ taxa) and genera ( $\geq 8$ taxa) in the Sutai Khairkhan Mountain Nature Reserve.

\begin{tabular}{lc|lc}
\hline Family & $\begin{array}{c}\text { Number } \\
\text { of taxa }\end{array}$ & Genus & $\begin{array}{c}\text { Number } \\
\text { of taxa }\end{array}$ \\
\hline Asteraceae & 48 & Potentilla & 17 \\
Fabaceae & 27 & Oxytropis & 13 \\
Rosaceae & 27 & Artemisia & 11 \\
Caryophyllaceae & 24 & Saussurea & 9 \\
Poaceae & 23 & Astragalus & 8 \\
Ranunculaceae & 14 & Pedicularis & 8 \\
\hline
\end{tabular}

Table 2. List of newly recorded species in the Mongolian Altai region.

\begin{tabular}{ll}
\hline Taxon & Family \\
\hline Astragalus brevifolius Ledeb. & Fabaceae \\
Carex coriophora Fisch. \& C.A.Mey. ex Kunth & Cyperaceae \\
Dasiphora parvifolia (Fisch. ex Lehm.) Juzz. & Rosaceae \\
Eritrichium alpinum Ovczinnikova & Boraginaceae \\
Koeleria macrantha (Ledeb.) Schult. & Poaceae \\
Leymus chinensis (Trin.) Tzvelev & Poaceae \\
Microula tibetica var. pratensis (Maxim.) W.T. Wang & Boraginaceae \\
Minuartia stricta (Sw.) Hiern & Caryophyllaceae \\
Persicaria minor (Huds.) Opiz & Polygonaceae \\
Potentilla tergemina Sojak & Rosaceae \\
Stellaria longifolia Muhl. ex Willd. & Caryophyllaceae \\
\hline
\end{tabular}

Table 3. Status of taxa, endemic, and threatened plants in the Sutai Khairkhan Mountain

\begin{tabular}{llllll}
\hline IUCN status & Family & Genus & Species & $\begin{array}{l}\text { Endemic \& Altai } \\
\text { Endemic species }\end{array}$ & $\begin{array}{l}\text { Threatened } \\
\text { species }\end{array}$ \\
\hline Total & 45 & 157 & 317 & 32 & 37 \\
Critically Endangered (CR) & 2 & 2 & 2 & - & 2 \\
Endangered (EN) & 6 & 8 & 11 & 5 & 11 \\
Vulnerable (VU) & 11 & 15 & 16 & 4 & 16 \\
Near threatened (NT) & 7 & 8 & 8 & 3 & 8 \\
\hline
\end{tabular}



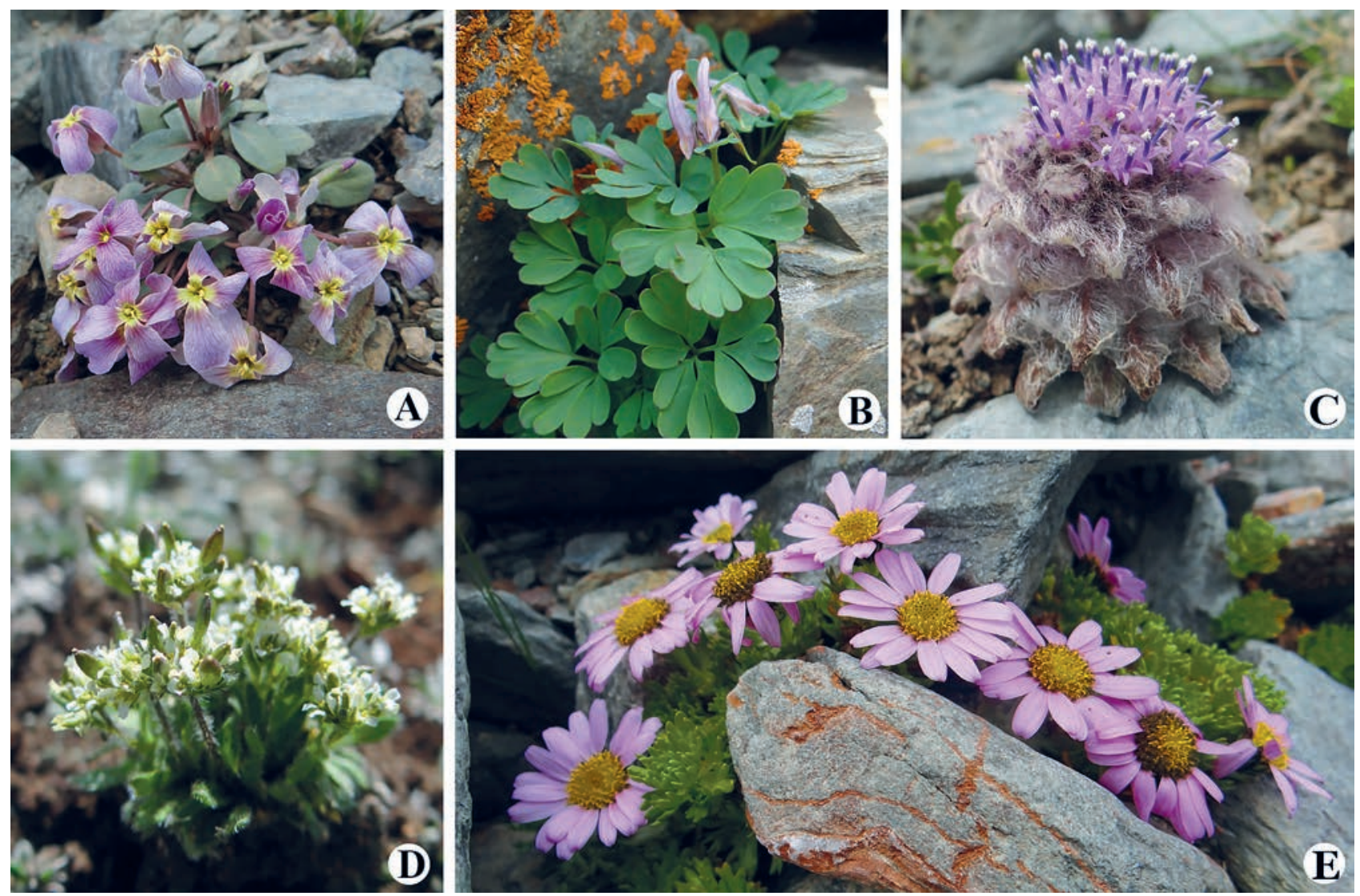

Figure 3 Examples of select rare and threatened plants from the Sutai Khairkhan Mountain Nature Reserve. A - Leiospora exscapa (C.A. Mey.) F. Dvořák; B - Corydalis inconspicua Bunge ex Ledeb.; C - Saussurea glacialis Herder; D - Draba altaica Bunge; E - Waldheimia tridactylites Kar. \& Kir. Photo: V. Gundegmaa

rare alpine plants. This complete checklist improves the current floristic knowledge of the SKMNR area, increases its conservation value, and provides a baseline for future research. This checklist is only foundational; additional studies should include vegetation surveys, hotspot richness surveys, and detailed distribution map of rare species in the SKMNR to further inform management and conservation strategies. Because alpine vegetation can move higher in elevation due to climate changes, and Mongolian's mountain taiga is predicted to be replaced by steppe species (Sainnemekh et al. 2022), monitoring the changes in plant community and rare species distribution, is needed. New distribution records of rare or endemic species can greatly affect our understanding of their abundance, threats, and genetics. Therefore, close studies of these species are needed to update the Mongolian Red List and inform the reserve's management to protect plant hotspots from overgrazing or other human disturbances.

\section{Taxonomic treatment}

Microula tibetica var. pratensis (Maxim.) W.T. Wang (Boraginaceae $)=$ Tretocarya pratensis Maxim.

In the literature, Tretocarya pratensis Maxim. was first recorded in the Khangai region of central Mongolia (Grubov 1982, Biazrov et al. 1989). In addition, we examined the herbarium specimens from all available herbaria which we found four herbarium specimens only from Mongolian Academy of Science (UBA), Mongolia. These four specimens were collected from the Khangai region of Mongolia between 1971 and 1976. Since 1976, there have been no record of wild populations and herbarium specimens in the country. Almost 40 years later, we found it on the Sutai Khairkhan Mountain, where there were less than 50 individuals within $1 \mathrm{~km}^{2}$ (Fig. 4D). In general, Tretocarya pratensis Maxim. was treated synonym of Microula tibetica var. pratensis (Maxim.) W.T. Wang by Zhu et al. (1995). According to Zhu et al. (1995), this varieties occurs only in the Qinghai, Xinjiang, and S. Xizang of China, but it is already distributed in Mongolia (Grubov 1982, Biazrov et al. 1989). In addition, Yu et al. (2012) confirmed two varieties Microula tibetica var. pratensis and M. tibetica var. tibetica are distinguished by nutlets and corolla limbs based on nutlet micro-morphology. Based on our collections, Mircoula tibetica var. pratensis is similar to $M$. tibetica var. tibetica but could easily be distinguished by the corolla limbs (1.2-1.8 mm wide; Fig. 4B) and nutlets with abaxial aperture (Fig. 4C) according to Zhu et al. (1995) and Yu et al. (2012).

Specimens examined. MONGOLIA. Khangai Region: Bayankhongor Province, Gurvanbulag sum, Shar Usnii Gol, 1971, D. Tsagaanmaam et al. s.n. (UBA); Bayankhongor Province, Erdenetsogt sum, Ovgor Khvren Mt, Namiin Gol, 22 July 1977, E. Ganbold et al. s.n. (UBA); Zavkhan province, Otgon sum, Otgontenger Mt, Chuluutiin gol, 3100 m a.s.l., 1974, (UBA); Khuvsgul Province, Arbulag sum, Sumber brigad, Dund gilaadiin am, 1976, D. Tsagaanmaam et al. s.n. (UBA). Mongolian Altai Region: Gobi-Altai Province, Tonkhil sum, Sutai Mt, 46³4'59.59"N 9337'55.32" E, 3281 m a.s.l., 16 July 2019, V. Gundegmaa et al. (UBU) (Fig. 5). 

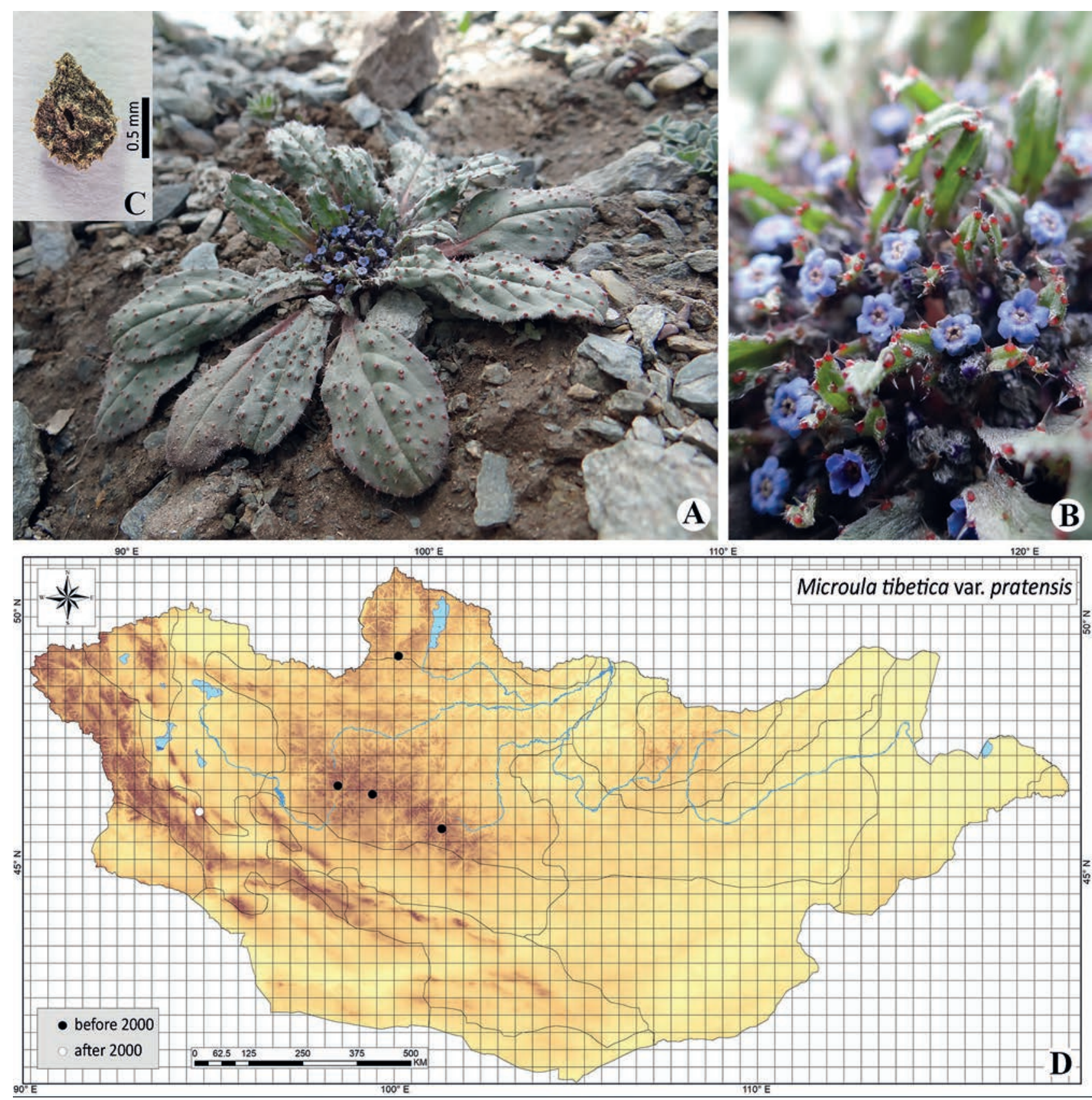

Figure 4 Microula tibetica var. pratensis (Maxim.) W.T. Wang. A - general habitat; B - flowers; C - abaxial part of nutlet with aperture; D distribution map (new wild locations in blue and herbarium collections in green). Photo: A, B - V. Gundegmaa, D - L. Jargal

\section{ACKNOWLEDGEMENTS}

Our gratitude goes to Dr. D. Narantsetseg and Dr. Ya. Shiilegmaa (Mongolian National University of Education) for supporting our field survey. We also give many thanks to Dr. Zhao Liqing (Normal University of Inner Mongolia) for crosscheckingplants and providing advice on our research. We extend our thanks to Mr. L. Jargal who took a photo of Microula tibetica nutlets. The research of Batlai Oyuntsetseg was supported by the National University of Mongolia (Grant No. P2021-4186). The research of Andrey Erst was supported by CSBG SB RAS (Grant No. AAAA-A21-121011290024-5).

\section{LITERATURE CITED}

Baasanmunkh, S., S. Takashi, B. Oyuntsetseg, K. Wesche, C.M. Ritz, K. Khaliunaa, J.Y. Kim, H.J. Jo, N. Batkhuu,
G.Y. Chung \& H.J. Choi 2019a. Contribution to the knowledge on the flora of Numrug Strictly Protected Area and some parts of East Mongolia. Journal of AsiaPacific Biodiversity 12:284-301.

Baasanmunkh, S., B. Oyuntsetseg, K. Oyundelger, K. Khaliunaa, M. Urgamal, N. Batkhuu, T. Shiga, G.Y. Chung \& H.J. Choi 2019b. Contribution to the knowledge on the flora of northern Mongolia. Journal of Asia-Pacific Biodiversity 12:643-660.

Baasanmunkh, S., N. Nyamgerel, G. Bayarmaa, B. Oyuntsetseg, K. Oyundelger \& H.J. Choi 2020. A new record of critically endangered Saussurea bogedaensis (Asteraceae) from Dzungarian Gobi, Mongolia. PhytoKeys 160:109-121.

Baasanmunkh, S., B. Oyunstsetseg, C. Oyundari, K. Oyundelger, M. Urgamal, D. Darikhand, N. Soninkhishig, D. Nyambayar, K. Khaliunaa, Z. Tsegmed, A.A. Kechaykin, A.I. Shmakov, A.S. Erst, N. Friesen, K. Wesche \& 

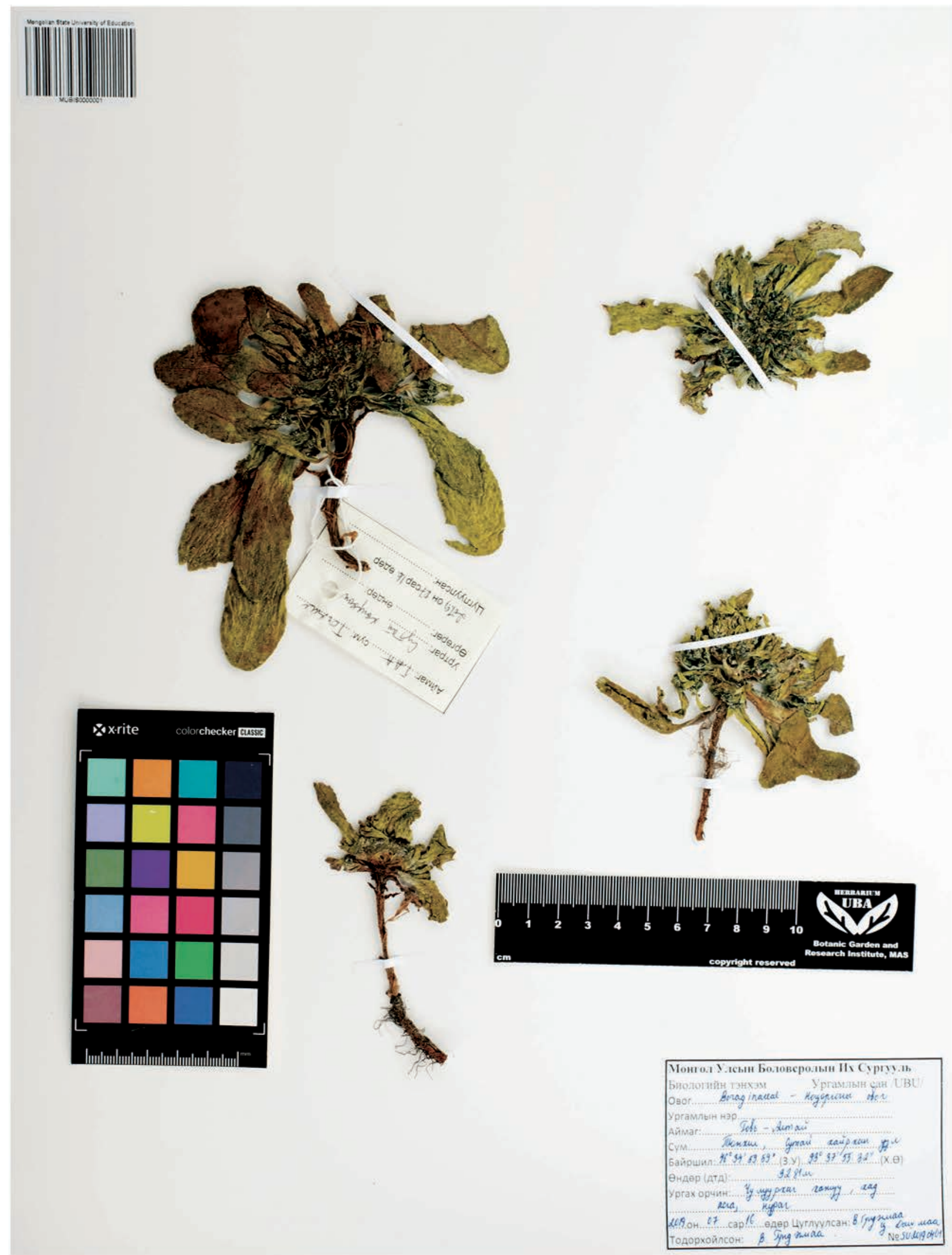

Figure 4 Microula tibetica var. pratensis (Maxim.) W.T. Wang. A - general habitat; B - flowers; C - abaxial part of nutlet with aperture; D distribution map (new wild locations in blue and herbarium collections in green). Photo: A, B - V. Gundegmaa, D - L. Jargal

H.J. Choi 2021a. The vascular plant diversity of Dzungarian Gobi in western Mongolia, with an annotated checklist. Phytotaxa 501(1):001-055.
Baasanmunkh, S., B. Oyunstsetseg, P. Efimov, Z. Tsegmed, S. Vandandorj, K. Oyundelger, M. Urgamal, A. Undruul, K. Khaliunaa, T. Namuulin \& H.J. Choi 2021b. Orchids of 
Mongolia: Taxonomy, species richness, and conservation status. Diversity 13: 302.

Baasanmunkh, S., M. Urgamal, B. Oyunstsetseg, A. Grabovskaya-Borodina, K. Oyundelger, V. Gundegmaa, Z. Tsegmed, A.A. Kechaykin, A.I. Pyak, L.Q. Zhao \& H.J. Choi 2021c. Updated checklist of vascular plants endemic to Mongolia. Diversity 13:619.

Baasanmunkh, S., B. Oyuntsetseg, Z. Tsegmed, K. Oyundelger, M. Urgamal, B. Gantuya, C. Javzandolgor, N. Nyambayar, P. Kosachev \& H.J. Choi 2022. Distribution of vascular plants in Mongolia - I part. Mongolian Journal of Biological Sciences 20:3-28.

Bekket, U., A.A. Kechaykin, I.Y. Yevdokimov, P.A. Kosachev \& A.I. Shmakov 2015. New findings about flora of West Mongolia. Acta Biologica Sibirica 1(2):132-139.

Biazrov, L.G., E. Ganbold, I.A. Gubanov \& N. Ulziikhutag 1989. Flora of Khangaya. Nauka, Lenningrad. 191 pp. (in Russian). [Бязров А.Г., ГанболА Э., Губанов И.А., УАьзихутаг Н. 1989. ФАора Хангая. А.: Наука. 191 с.].

eFloras. 2008. Flora of China. Missouri Botanical Garden and Harvard University Herbaria, St. Louis, MO, US and Cambridge, MA, US.

Erst, A.S., A.Yu. Nikulin, V.Yu. Nikulin, A.L. Ebel, E.V. Zibzeev, M.T. Sharples, S. Baasanmunkh, M.V. Olonova, A.I. Pyak, I.I. Gureeva, T.V. Erst, A.A. Kechaykin, A. Luferov, M. Nobis, L. Lian \& W. Wang 2021. Distribution analysis, updated checklist, and DNA barcodes of the endemic vascular flora of the Altai Mountains, a biodiversity hotspot in Siberia. Systematics and Biodiversity (in press).

German, D.A. 2015. Cruciferae (Brassicaceae): Alternative treatment for the "Conspectus of the vascular plants of Mongolia". Turczaninowia 18(2):39-67.

Grubov, V.I. 1982. Key to the vascular plants of Mongolia (with an atlas). Nauka, Leningrad, 443 pp. (in Russian). [Tpyбов В.И. 1982. Определитель сосудистых растений Монголии. А.: Наука. 443 с.].

Gubanov, I.A. 1996. Conspectus of the flora of Outer Mongolia (vascularplants). Valang, Moscow, 136 pp. (in Russian). [Tyбанов И.А. 1996. Конспект флоры Внешней Монгомии (сосудистые растения). М.: Валанг. 136 с.].

Gundegmaa, V. \& T. Munkh-Erdene 2018. Field guide to Alpine flowers of Mongolian Altai. Soyombo printing, Ulaanbaatar, $232 \mathrm{pp}$.

Gundegmaa, V., T. Munkh-Erdene, M. Urgamal \& C. Bilegtmandakh 2018. The rare and threatened species of plant diversity at climate change of Mongolian Altai. Proceeding of the Mongolian Academy of Sciences 34:168-177.

Neuffer, B., B. Oyuntsetseg, Z. Schamsran, N. Friesen \& H. Hurka 2003. Contribution to the knowledge of the Flora of the Mongolian Altai. Feddes Repertorium 114:358-371.

Nobis, M., E. Klichowska, A. Terlević, A. Wróbel, A. Erst, R. Hrivnák, A.L. Edel et al. 2019. Contribution to the flora of Asian and European countries: new national and regional vascular plant records, 8. Botany Letters 166(2):163-188.

Kamelin, R.V. 2005. New Altai flora. Brief characteristics of natural conditions and vegetation of Altai mountain country. In: Altai flora, vol. 1 (R.V. Kamelin, ed.), pp. 6897, AzBuka, Barnaul (in Russian). [Камелин P.B. 2005. Новая флора А^тая. Краткий очерк природных условий и растительного покрова А^тайской горной страны / / ФАора Алтая / отв. реА. Р.В. Камелин. Барнаул: АзБука. Т. 1. С. 7-97].

Olonova, M.V., D.Y. Zhang, S.M. Duan, S.M. Yin \& B.R. Pan 2010. Rare and endangered plant species of the Chinese Altai Mountains. Journal of Arid Land 2:222-230.
Ovchinnikova, S.V. 2020. Three new species of the genus Craniospermum (Boraginaceae) from Mongolia. Turczaninowia 23(2):108-119.

Oyuntsetseg, B., S. Baasanmunkh, D. Nyambayar, N. Batkhuu, C.H. Lee, C.K. Sun, G.Y. Chung \& H.J. Choi 2018. The conservation status of 100 rare plants in Mongolia. Pocheon, Korea, 232 pp.

Oyuntsetseg, B., S. Baasanmunkh, K. Oyundelger, O. Munkhzul, J.Y. Kim, H.J. Cho, B. Nyam-Osor, G.Y. Chung \& H.J. Choi. 2017. Contribution to the knowledge on the flora of Munkhkhairkhan mountain area, Mongolia. Journal of Asia-Pacific Biodiversity 10:573-582.

POWO. 2019. Plants of the World Online. Facilitated by the Royal Botanic Gardens, Kew. Published on the Internet: http:// www.plantsoftheworldonline.org/. Retrieved: 01.12 .2020

Pyak, A.I. \& E.A. Pyak 2019. A new species of Astragalus section Laguropsis (Fabaceae: Galegeae) from a cross border highland region of Altai Mountains in Russia and Mongolia. Phytotaxa 414(4):194-198.

Pyak, E.A., A.I. Pyak \& V.V. Madyka 2020. Saussurea odorata (Asteraceae), a new species from western Mongolia. Phytotaxa 470:235-242.

Pyak, A.I., S.C. Shaw, A.L. Ebel, A.A. Zverev, J.G. Hodgson, B.D. Wheeler, K.J. Gaston et al. 2008. Endemic plants of the Altai Mountain country. Wild Guides Ltd., UK, 368 pp.

Sainnemekh, S., I.C. Barrio, B. Densambuu, B. Bestelmeyer \& A.L. Aradottir 2022. Rangeland degradation in Mongolia: A systematic review of the evidence. Journal of Arid Environments 196:104654

Shiga, T., K. Khaliunaa, S. Baasanmunkh, B.Oyuntsetseg, S. Midorikawa \& H.J. Choi 2020. New Mongolian records of two genera, seven species, and two hybrid nothospecies from Khar-Us Lake and its associated wetlands. Journal of Asia-Pacific Biodiversity 13: 443-453.

Urgamal, M., B. Oyuntsetseg, D. Nyambayar \& C. Dulamsuren 2014. Conspectus of the vascular plants of Mongolia. Admon Printing Press, Ulaanbaatar, 334 pp.

Urgamal, M., V. Gundegmaa, S. Baasanmunkh, B. Oyuntsetseg, D. Darikhand \& T. Munkh-Erdene 2019a. Additions to the Vascular Flora of Mongolia - IV. Proceeding of the Mongolian Academy of Sciences 59(2):14-53.

Urgamal, M., B. Oyuntsetseg, R. Tungalag, V. Gundegmaa, C. Oyundari, T. Tserendulam, T. Munkh-Erdene 2019b. Red List of Mongolia 2. Ulaanbaatar, 230 pp. (in Mongolian). ГУргамах М., Оюнцацаг Б., Тунгалаг Р., Гундэгмаа В., Оюндарь Ч., Цэрэндулам Ц., Менх-Эрдэнэ Т. 2019. Монгол орны Ургамлын У аан данс. Хоёрдугаар цуврал. УАаан-Баатар. 230 х.].

Vaganov, A.V., A.I. Shmakov \& P.D. Gudkova 2019. Global data on biodiversity of the Altai mountain country, presented in the world's scientific depositories. Acta Biologica Sibirica 5(2):95-101.

Yu, W.T., F.M. Jacques, S.T. Chen \& Z.K. Zhou 2012. Nutlet micro-morphology of the genus Microula (Boraginaceae) from the Qinghai-Tibetan Plateau, and its systematic implications. Nordic Journal of Botany 30(5):596-612.

Zhao, L.Q., X. Ri, Alatanzhula, V. Gundegmaa, X.G. Qiao \& C. Mungunchimeg 2019. Stipa khovdensis (Poaceae), a new species, and a checklist of Stipa s. stricto from Mongolia. Annales Botanici Fennici 56:95-99.

Zhu, G.L., H. Riedl \& V.R. Kamelin 1995. Microula Benth. In: Flora of China. Vol. 64. (Flora of China Editorial Committee, ed.), pp. 392-401, Miss. Bot. Gard. Press. 
Appendix 1. A checklist of vascular plants in the SKM NR, with the growth form, status, elevation, phytogeographical regions, and herbarium code for each species. (Growth form: H - herb, T - tree, S - shrub, SS - subshrub; Status: E Endemic, AE - Altai Endemic, CR - Critically Endangered, EN - Endangered, VU - Vulnerable, NT - Near Threatened. Distribution of phytogeographical regions based on Urgamal et al. (2014) and new distribution records marked by +): $1-$ Khuvsgul, 2 - Khentei, 3 - Khangai, 4 - Mongolian Dauria, 5 - Foothills of Great Khingan, 6 - Khovd, 7 - Mongolian Altai, 8 - Middle Khalkh, 9 - East Mongolia, 10 - Depression of Great Lakes, 11 - Valley of Lakes, 12 - East Gobi, 13 -Gobi Altai, 14 - Dzungarian Gobi, 15 - Transaltai Gobi, and 16 - Alashan Gobi.

\begin{tabular}{|c|c|c|c|c|c|}
\hline Taxon & $\begin{array}{l}\text { Growth } \\
\text { form }\end{array}$ & $\begin{array}{l}\text { IUCN } \\
\text { status }\end{array}$ & $\begin{array}{l}\text { Elevation, } \\
\text { m a.s.1 } \\
\text { (low to high) }\end{array}$ & Region & $\begin{array}{l}\text { Herbarium } \\
\text { code }\end{array}$ \\
\hline \multicolumn{6}{|l|}{ FERNS AND FERN ALLIES } \\
\hline \multicolumn{6}{|l|}{ Aspleniaceae } \\
\hline Asplenium altajense (Kom.) Grubov & $\mathrm{H}$ & & $2100-2500$ & $1,3,4,7,10,13$ & SU20190306 \\
\hline \multicolumn{6}{|l|}{ Cystopteridaceae } \\
\hline Cystopteris fragilis (L.) Bernh. & $\mathrm{H}$ & & $2600-3300$ & $1-10,13,14,15$ & SU20190310 \\
\hline \multicolumn{6}{|l|}{ Ophioglossaceae } \\
\hline Botrycbium lunaria (L.) Sw. & $\mathrm{H}$ & & $2800-3000$ & $1,2,3,4,5,7,9$ & SU20200507 \\
\hline \multicolumn{6}{|l|}{ Woodsiaceae } \\
\hline Woodsia ilvensis (L.) R. Br. & $\mathrm{H}$ & & $2600-3000$ & $1,2,3,4,7,8,9$ & SU20190307 \\
\hline Woodsia glabella $\mathrm{R}$. Br. & $\mathrm{H}$ & & $2600-3100$ & $1,7,10$ & SU20190311 \\
\hline \multicolumn{6}{|l|}{ GYMNOSPERMS } \\
\hline \multicolumn{6}{|l|}{ Pinaceae } \\
\hline Larix sibirica Ledeb. & $\mathrm{T}$ & & $2000-2500$ & $1,2,3,4,6,7,8,10,14$ & SU20200220 \\
\hline \multicolumn{6}{|l|}{ Cupressaceae } \\
\hline Juniperus pseudosabina Fisch. \& C.A.Mey. & S & $\mathrm{EN}$ & $2600-3000$ & $1,2,3,4,7,8,13$ & SU20140601 \\
\hline \multicolumn{6}{|l|}{ Ephedraceae } \\
\hline Ephedra monosperma J.G.Gmel. ex C.A.Mey. & SS & & $1500-2500$ & $1-8,10,12,13$ & SU20190204 \\
\hline \multicolumn{6}{|l|}{ ANGIOSPERMS } \\
\hline \multicolumn{6}{|l|}{ Amaranthaceae } \\
\hline Blitum virgatum L. & $\mathrm{H}$ & & $1600-2000$ & $3,4,6,7,12-15$ & SU20200908 \\
\hline Chenopodium album $\mathrm{L}$. & $\mathrm{H}$ & & $1500-2100$ & $1-16$ (all regions) & $\begin{array}{l}\text { SU20200610, } \\
\text { SU20200912 }\end{array}$ \\
\hline Chenopodium frutescens C.A.Mey. & SS & $\mathrm{AE}$ & $1500-2000$ & $6,7,10$ & SU20140310 \\
\hline Grubovia dasyphylla (Fisch. \& C.A. Mey.) Freitag \& G. Kadereit & $\mathrm{H}$ & & $1500-2200$ & $3-16$ & SU20200903 \\
\hline Krascheninnikovia ceratoides (L.) Gueldenst. & SS & & 1500 & $1,3,4,6,7,8,10-16$ & $\begin{array}{l}\text { SU20190101, } \\
\text { SU20200906 }\end{array}$ \\
\hline Salsola collina Pall. & $\mathrm{H}$ & & $1700-2300$ & $2-15$ & SU20200907 \\
\hline Suaeda tschujensis Lomon. \& Freitag & $\mathrm{H}$ & $\mathrm{AE}$ & $1500-2000$ & 6,7 & SU20140206 \\
\hline \multicolumn{6}{|l|}{ Amaryllidaceae } \\
\hline Allium altaicum Pall. & $\mathrm{H}$ & VU & $2800-3050$ & $1,2,3,6,7,8,10,13,14$ & SU20140504 \\
\hline Allium mongolicum Regel & $\mathrm{H}$ & & $1500-2100$ & $3,4,6-16$ & SU20200802 \\
\hline Allium polyrbizum Turcz. ex Regel & $\mathrm{H}$ & & $1600-2000$ & $1,2,3,4,7-13,15,16$ & SU20200803 \\
\hline Allium prostratum Trevir. & $\mathrm{H}$ & & $1800-2300$ & $1-13$ & SU20190106 \\
\hline Allium pumilum Vved. & $\mathrm{H}$ & $\mathrm{AE}$ & $2000-2600$ & $6,7,14$ & SU20190105 \\
\hline \multicolumn{6}{|l|}{ Apiaceae } \\
\hline Bupleurum bicaule Helm & $\mathrm{H}$ & & $1500-2300$ & $1,2,3,4,6-13$ & $\begin{array}{l}\text { SU20200602, } \\
\text { SU20200709 }\end{array}$ \\
\hline Bupleurum mongolicum V.M. Vinogr. & $\mathrm{H}$ & & $3000-3350$ & $7,13,14$ & SU20201510 \\
\hline Carum carvi $\mathrm{L}$. & $\mathrm{H}$ & & $1800-3000$ & $1-5,7,8,9,10,14$ & SU20200215 \\
\hline Ferulopsis bystrix (Bunge) Pimenov & $\mathrm{H}$ & & $1900-2700$ & $2,3,4,6-11,13,15$ & $\begin{array}{l}\text { SU20190108, } \\
\text { SU20200101, } \\
\text { SU20200206 }\end{array}$ \\
\hline Neogaya simplex (L.) Meisn. & $\mathrm{H}$ & & $2100-3000$ & $1,2,3,4,6,7,13,14$ & SU20190504 \\
\hline Ostericum tenuifolium (Pall. ex Schult.) Y.C. Chu & $\mathrm{H}$ & & $2200-3000$ & $1,2,3,4,6-10,13$ & $\begin{array}{l}\text { SU20190508, } \\
\text { SU20200503 }\end{array}$ \\
\hline Seseli buchtormense (Fisch. ex Hornem.) W.D.J. Koch & $\mathrm{H}$ & & $2100-3000$ & 7,14 & SU20201405 \\
\hline Seseli condensatum (L.) Rchb. f. & & & $2100-3000$ & $1,2,3,6,7,8,10,14$ & SU20201310 \\
\hline \multicolumn{6}{|l|}{ Asteraceae } \\
\hline Ajania grubovii Muldashev & SS & $\mathrm{E}$ & 1500 & 7,14 & SU20140102 \\
\hline Artemisia argyrophylla Ledeb. & SS & $\mathrm{AE}$ & $1800-2100$ & $1,3,6,7,13,15$ & SU20200410 \\
\hline Artemisia campestris subsp. borealis (Pall.) H.M.Hall \& Clem & $\mathrm{H}$ & & $2000-2600$ & $1,2,3,4,6,7,10,13$ & $\begin{array}{l}\text { SU20190203, } \\
\text { SU20200910, } \\
\text { SU20201002 }\end{array}$ \\
\hline Artemisia dracunculus $\mathrm{L}$. & $\mathrm{H}$ & & $1500-2500$ & $1-15$ & $\begin{array}{l}\text { SU20140202, } \\
\text { SU20140304, } \\
\text { SU20190102, } \\
\text { SU20190201, } \\
\text { SU20201001 }\end{array}$ \\
\hline $\begin{array}{l}\text { Artemisia dracunculus var. pamirica (C.Winkl.) Y.R.Ling \& } \\
\text { Humphries }\end{array}$ & $\mathrm{H}$ & & $1800-2500$ & $3,6,7,10,11,12,13$ & $\begin{array}{l}\text { SU20200204, } \\
\text { SU20200302, } \\
\text { SU20200404 }\end{array}$ \\
\hline
\end{tabular}


Appendix 1. Continued

\begin{tabular}{|c|c|c|c|c|c|}
\hline Taxon & $\begin{array}{l}\text { Growth } \\
\text { form }\end{array}$ & $\begin{array}{l}\text { IUCN } \\
\text { status }\end{array}$ & $\begin{array}{l}\text { Elevation, } \\
\text { m a.s.1 } \\
\text { (low to high) }\end{array}$ & Region & $\begin{array}{l}\text { Herbarium } \\
\text { code }\end{array}$ \\
\hline Artemisia frigida Wild. & SS & & $1500-2100$ & 1-16 (all regions) & $\begin{array}{l}\text { SU20190203, } \\
\text { SU20190202, } \\
\text { SU20200402 }\end{array}$ \\
\hline Artemisia laciniata Wild. & $\mathrm{H}$ & & $1600-2500$ & $1-5,7,8,9,10,12,14$ & $\begin{array}{l}\text { SU20190202, } \\
\text { SU20200304 }\end{array}$ \\
\hline Artemisia macrocephala Jacquem. ex Besser & $\mathrm{H}$ & & $1500-2600$ & $1-16$ (all regions) & $\begin{array}{l}\text { SU20190205, } \\
\text { SU20200203, }\end{array}$ \\
\hline Artemisia mongolica (Fisch. ex Besser) Nakai & $\mathrm{H}$ & & $1600-2000$ & $1-15$ & SU20190301 \\
\hline Artemisia pycnorrbiza Ledeb. & $\mathrm{H}$ & & $1500-2500$ & $1-4,6,7,8,10,11,13,14$ & $\begin{array}{l}\text { SU20190103, } \\
\text { SU20200305 }\end{array}$ \\
\hline Artemisia stechmanniana Besser & SS & LC & $1500-2500$ & $2,3,6-15$ & SU20200601 \\
\hline Artemisia xerophytica Krasch. & S & & $2000-3000$ & $6,7,8,10-16$ & SU20190104 \\
\hline Askellia pygmaea (Ledeb.) Sennikov & $\mathrm{H}$ & VU & $2900-3250$ & $1,3,6,7$ & $\begin{array}{l}\text { SU20190305, } \\
\text { SU20201502 }\end{array}$ \\
\hline Aster alpinus $\mathrm{L}$. & $\mathrm{H}$ & & $1600-2700$ & $1-10,13$ & SU20200702 \\
\hline Aster altaicus Willd. & $\mathrm{H}$ & & $1500-2100$ & $1,2,3,4,6,7,8,10,12-16$ & SU20140306 \\
\hline Aster flaccidus subsp. flaccidus & $\mathrm{H}$ & & $2100-2500$ & $1,2,3,4,6,7$ & SU20200202 \\
\hline Asterothamnus centrali-asiaticus Novopokr. & SS & LC & $1800-2000$ & $7,8,9,11-16$ & SU20140406 \\
\hline Asterothamnus heteropappoides Novopokr. & SS & $\mathrm{AE}, \mathrm{NT}$ & Г1500-1800 & $6,7,10,14$ & SU20140502 \\
\hline Cirsium esculentum (Siev.) C.A.Mey. & $\mathrm{H}$ & & $2000-2800$ & $1,2,3,4,6-11,14$ & SU20201302 \\
\hline Crepidiastrum tenuifolium (Willd.) Sennikov & $\mathrm{H}$ & & $1800-2300$ & $1-11,13,14$ & SU20200603 \\
\hline Crepis chrysantha (Ledeb.) Turcz. & $\mathrm{H}$ & & $3000-3300$ & $1,2,3,6,7,10$ & SU20190403 \\
\hline Doronicum turkestanicum Cavill. & $\mathrm{H}$ & & $3050-3200$ & $3,7,14$ & SU20190311 \\
\hline Erigeron altaicus Popov & $\mathrm{H}$ & $\mathrm{AE}$ & $2100-2600$ & 7,14 & $\begin{array}{l}\text { SU20190308, } \\
\text { SU20200301 }\end{array}$ \\
\hline Erigeron eriocalyx (Ledeb.) Vierh. & $\mathrm{H}$ & & $2100-2500$ & $1,2,3,6,7,13$ & SU20200303 \\
\hline Erigeron petiolaris Vierh. & $\mathrm{H}$ & & $2200-2500$ & 3,7 & SU20200306 \\
\hline $\begin{array}{l}\text { Leontopodium nanum (Hook.f. \& Thomson ex C.B.Clarke) Hand.- } \\
\text { Mazz. }\end{array}$ & $\mathrm{H}$ & & $2100-3200$ & 7,16 & $\begin{array}{l}\text { SU20140503, } \\
\text { SU20200403 }\end{array}$ \\
\hline Leontopodium ochroleucum Beauverd & $\mathrm{H}$ & & $2100-3300$ & $1,2,3,6,7,13$ & $\begin{array}{l}\text { SU20140402, } \\
\text { SU20200308 }\end{array}$ \\
\hline Saussurea glacialis Herder & $\mathrm{H}$ & $\mathrm{EN}$ & $<3500$ & $3,6,7,13$ & $\begin{array}{l}\text { SU20190501, } \\
\text { SU20201505 }\end{array}$ \\
\hline Saussurea latifolia Ledeb. & $\mathrm{H}$ & VU & $1600-2500$ & 3,7 & SU20190107 \\
\hline Saussurea leucophylla Schrenk & $\mathrm{H}$ & & $2000-2200$ & $1,3,6,7,13$ & SU20190109 \\
\hline Saussurea orgaadayi Khanm. \& Krasnob. & $\mathrm{H}$ & $\mathrm{AE}, \mathrm{EN}$ & J $3000-3300$ & 7 & $\begin{array}{l}\text { SU20140505, } \\
\text { SU20201203 }\end{array}$ \\
\hline Saussurea pricei N.D.Simpson & $\mathrm{H}$ & & $2000-3000$ & $3,6,7,8,10,11,13,14$ & SU20140305 \\
\hline Saussurea pseudoalpina N.D.Simpson & $\mathrm{H}$ & & $2600-3000$ & $1,2,3,6,7,13,14$ & SU20201705 \\
\hline Saussurea saichanensis Kom. ex Lipsch. & $\mathrm{H}$ & $\mathrm{E}$ & $2000-3000$ & $1,2,3,6,7,13,14$ & SU20140501 \\
\hline Saussurea schanginiana (Wydler) Fisch. ex Herder & $\mathrm{H}$ & & $3000-3300$ & $1,2,3,6,7,13$ & SU20140405 \\
\hline Saussurea subacaulis (Ledeb.) Serg. & $\mathrm{H}$ & VU & $3000-3300$ & $1,3,6,7,13$ & SU20140302 \\
\hline Scorzonera ikonnikovii Lipsch. \& Krasch. & $\mathrm{H}$ & & $1800-2300$ & $3,6-15$ & SU20200703 \\
\hline Senecio dubitabilis C.Jeffrey \& Y.L.Chen & $\mathrm{H}$ & & $1800-2200$ & $2,3,7,8,10-15$ & SU20200608 \\
\hline $\begin{array}{l}\text { Tanacetum changaicum (Krasch. ex Grubov) K.Bremer \& } \\
\text { Humphries }\end{array}$ & $\mathrm{H}$ & $\mathrm{E}, \mathrm{EN}$ & $2900-3300$ & $3,7,10$ & $\begin{array}{l}\text { SU20190312, } \\
\text { SU20201210 }\end{array}$ \\
\hline Tanacetum lanuginosum Sch.Bip. \& Herder & $\mathrm{H}$ & & $3000-3300$ & $1,6,7,13$ & SU20190313 \\
\hline Tanacetum pulchrum (Ledeb.) Sch.Bip. & $\mathrm{H}$ & VU & $3000-3300$ & $3,7,13$ & SU20140409 \\
\hline Taraxacum ceratophorum (Ledeb.) DC. & $\mathrm{H}$ & & $2300-3000$ & $3,6,7$ & SU20201406 \\
\hline Taraxacum dissectum (Ledeb.) Ledeb. & $\mathrm{H}$ & & $2000-3000$ & $1,2,3,4,6-10,12,13$ & SU20200106 \\
\hline Taraxacum junatovii Tzvelev & $\mathrm{H}$ & $\mathrm{E}$ & $2600-3000$ & $3,7,13,14$ & SU20140306 \\
\hline Taraxacum lyratum (Ledeb.) DC. & $\mathrm{H}$ & $\mathrm{AE}$ & $2600-3000$ & $1,3,6,7,13$ & SU20201304 \\
\hline Tephroseris integrifolia (L.) Holub & $\mathrm{H}$ & & $3000-3300$ & $1,2,3,4,6,7,8,9,13$ & SU20190314 \\
\hline Tephroseris pricei (N.D.Simpson) Holub & $\mathrm{H}$ & & $1800-2000$ & $1,3,6,7,13,14$ & SU20190315 \\
\hline Waldheimia tridactylites Kar. \& Kir. & $\mathrm{H}$ & & $<3500$ & $1,3,6,7,13$ & $\begin{array}{l}\text { SU20190403, } \\
\text { SU20201404 }\end{array}$ \\
\hline \multicolumn{6}{|l|}{ Boraginaceae } \\
\hline Amblynotus rupestris (Georgi) Popov & $\mathrm{H}$ & & $2200-3000$ & $1-9,13$ & SU20200309 \\
\hline Craniospermum canescens DC. & $\mathrm{H}$ & $\mathrm{AE}, \mathrm{VU}$ & J $2000-2500$ & $3,7,13,14$ & SU20140204 \\
\hline Eritrichium alpinum Ovczinnikova & $\mathrm{H}$ & $\mathrm{AE}, \mathrm{VU}$ & J $2100-2800$ & $6,7+$ & SU20200310 \\
\hline Eritrichium pauciflorum DC. & $\mathrm{H}$ & & $2100-2600$ & $1-8,13$ & SU20200312 \\
\hline Microula tibetica var. pratensis (Maxim.) W.T.Wang & $\mathrm{H}$ & & $3000-3300$ & $3,7+$ & SU20190401 \\
\hline Myosotis alpestris F.W.Schmidt & $\mathrm{H}$ & & $2500-3300$ & $1,2,3,4,6,7,9,14$ & SU20200313 \\
\hline Myosotis asiatica (Vestergr.) Schischk. \& Serg. & $\mathrm{H}$ & & $2100-2800$ & $1,2,3,4,6,7,9,14$ & SU20200505 \\
\hline Myosotis austrosibirica O.D.Nikif. & $\mathrm{H}$ & & $2100-2800$ & 7,13 & SU20190309 \\
\hline
\end{tabular}


Appendix 1. Continued

\begin{tabular}{|c|c|c|c|c|c|}
\hline Taxon & $\begin{array}{l}\text { Growth } \\
\text { form }\end{array}$ & $\begin{array}{l}\text { IUCN } \\
\text { status }\end{array}$ & $\begin{array}{l}\text { Elevation, } \\
\text { m a.s.1 } \\
\text { (low to high) }\end{array}$ & Region & $\begin{array}{l}\text { Herbarium } \\
\text { code }\end{array}$ \\
\hline \multicolumn{6}{|l|}{ Brassicaceae } \\
\hline Dontostemon senilis Maxim. & $\mathrm{H}$ & & $1500-2100$ & $6,7,8,10-16$ & SU20200607 \\
\hline Draba altaica (C.A.Mey.) Bge & $\mathrm{H}$ & VU & $3000-3300$ & $6,7,10$ & SU20201407 \\
\hline Draba cana Rydb. & $\mathrm{H}$ & & $2100-2500$ & $1,2,3,4,6,7,13$ & SU20200313 \\
\hline Draba lanceolata Royle & $\mathrm{H}$ & & $2600-3300$ & $1,2,3,4,6,7,13$ & SU20190316 \\
\hline Draba nemorosa $\mathrm{L}$. & $\mathrm{H}$ & & $2600-3300$ & $1-10,13$ & SU20190502 \\
\hline Draba oreades Schrenk & $\mathrm{H}$ & & $<3500$ & $1,3,6,7,13$ & $\begin{array}{l}\text { SU20190503, } \\
\text { SU20201506 }\end{array}$ \\
\hline Leiospora exscapa (C.A.Mey.) F.Dvorák & $\mathrm{H}$ & $\mathrm{AE}$ & $<3500$ & $1,6,7$ & SU20190402 \\
\hline Pachyneurum grandiflorum (C.A.Mey.) Bunge & $\mathrm{H}$ & $\mathrm{AE}$ & $3000-3300$ & $1,3,6,7,13$ & SU20190408 \\
\hline Ptilotrichum canescens (DC.) C.A.Mey. & $\mathrm{H}$ & & $1500-2100$ & $1-4,6-9,11,12,13,15,16$ & SU20140103 \\
\hline Smelowskia alba (Pall.) Regel & $\mathrm{H}$ & & $3000-3350$ & $1,3,4,6,7,10,13$ & SU20190307 \\
\hline Smelowskia altaica (Pobed.) Botsch & $\mathrm{H}$ & $\mathrm{AE}, \mathrm{VU}$ & J $3000-3300$ & 6,7 & SU20190303 \\
\hline Smelowskia calycina (Stephan ex Willd.) C.A.Mey. & $\mathrm{H}$ & & $<3500$ & $1,3,6,7,13,14$ & SU20190401 \\
\hline \multicolumn{6}{|l|}{ Caprifoliaceae } \\
\hline Lonicera caerulea subsp. altaica (Pall.) Gladkova & $\mathrm{S}$ & & $2200-2600$ & $1,2,3,4,6,7,13,14$ & SU20140401 \\
\hline Lonicera microphylla Willd. ex Schult. & $\mathrm{S}$ & & $2000-2800$ & $3,6,7,9,10,13,14,16$ & SU20140307 \\
\hline Valeriana petrophila Bunge & $\mathrm{H}$ & & $3000-3300$ & $1,3,6,7,13$ & SU20190304 \\
\hline \multicolumn{6}{|l|}{ Caryophyllaceae } \\
\hline Cerastium arvense $\mathrm{L}$. & $\mathrm{H}$ & & $2100-2600$ & $1-7,9,10,13,14$ & $\begin{array}{l}\text { SU20200510, } \\
\text { SU20200511 }\end{array}$ \\
\hline Cerastium lithospermifolium Fisch. & $\mathrm{H}$ & & $<3500$ & $1,3,6,7,10,13$ & $\begin{array}{l}\text { SU20190406, } \\
\text { SU20190407, } \\
\text { SU20201403 }\end{array}$ \\
\hline Cerastium pusillum Ser. & $\mathrm{H}$ & & $2600-3300$ & $1,2,3,6,7$ & SU20201403 \\
\hline Cherleria biflora (L.) A.J.Moore \& Dillenb. & $\mathrm{H}$ & & $2500-3300$ & $1,2,3,6,7$ & SU20201702 \\
\hline Dianthus chinensis L. & $\mathrm{H}$ & & $2100-2500$ & $1-11,13$ & SU20140105 \\
\hline Dianthus repens Willd. & $\mathrm{H}$ & & $2500-2800$ & 6,7 & SU20140207 \\
\hline Dianthus superbus L. & $\mathrm{H}$ & & $2100-2500$ & $1-10,13$ & SU20200504 \\
\hline Dichodon cerastoides (L.) Rchb. & $\mathrm{H}$ & & $2600-3000$ & $1-7,10,14$ & SU20200508 \\
\hline Eremogone androsacea (Grubov) Ikonn. & $\mathrm{H}$ & $\mathrm{EN}$ & $2200-3000$ & 13,7 & SU20200411 \\
\hline Eremogone meyeri (Fenzl) Ikonn. & $\mathrm{H}$ & & $2300-3000$ & $2,3,4,6,7,9,10,12,13$ & SU20140105 \\
\hline Eremogone mongholica (Schischk.) Ikonn. & $\mathrm{H}$ & $\mathrm{AE}, \mathrm{EN}$ & $\mathrm{N} 2500-3000$ & 7 & SU20200413 \\
\hline Heterochroa desertorum Bunge & $\mathrm{H}$ & & $1500-2300$ & $1,2,3,4,6-13,16$ & SU20190113 \\
\hline Minuartia stricta (Sw.) Hiern & $\mathrm{H}$ & NT & $2500-3200$ & $1,2,3,7+$ & $\begin{array}{l}\text { SU20190303, } \\
\text { SU202016 }\end{array}$ \\
\hline Sabulina verna (L.) Rchb. & $\mathrm{H}$ & & $2500-3250$ & $1,2,3,6,7,14$ & SU20201408 \\
\hline Silene amoena $\mathrm{L}$. & $\mathrm{H}$ & & $2100-3300$ & $6,7,10,14$ & SU20190315 \\
\hline Silene chamarensis Turcz. & $\mathrm{H}$ & & $2100-3300$ & $1,2,3,6,7,9,10,12,13$ & SU20190114 \\
\hline Silene songarica (Fisch., C.A.Mey. \& Avé-Lall.) Bocquet & $\mathrm{H}$ & & $2600-3000$ & $1-7,9,12,13$ & $\begin{array}{l}\text { SU20190317, } \\
\text { SU20201204 }\end{array}$ \\
\hline Silene uralensis subsp.apetala (L.) Bocquet. & $\mathrm{H}$ & & $2000-3000$ & $1,2,3,6,7,10,13,14$ & SU20140303 \\
\hline Stellaria brachypetala Bunge & $\mathrm{H}$ & & $2000-2600$ & $3-7,9,11,13,14$ & SU20200501 \\
\hline Stellaria dichotoma $\mathrm{L}$. & $\mathrm{H}$ & $\mathrm{LC}$ & $1800-2500$ & $1-14$ & SU20200902 \\
\hline Stellaria imbricata Bunge & $\mathrm{H}$ & $\mathrm{AE}$ & $2000-2500$ & $6,7,14$ & SU20190318 \\
\hline Stellaria longifolia Muhl. ex Willd. & $\mathrm{H}$ & & $2100-2800$ & $1,2,3,4,5,7+, 9$ & SU20200509 \\
\hline Stellaria palustris Ehrh. ex Hoffm. & $\mathrm{H}$ & & $2100-2500$ & $2,3,7,9$ & SU20200319 \\
\hline Stellaria pulvinata Grubov & $\mathrm{H}$ & $\mathrm{AE}, \mathrm{VU}$ & U 2000-2800 & 6,7 & SU20190207 \\
\hline \multicolumn{6}{|l|}{ Crassulaceae } \\
\hline Orostachys spinosa (L.) Sweet & $\mathrm{H}$ & & $1500-2200$ & $1,2,3,4,6-15$ & SU20200102 \\
\hline Rhodiola quadrifida (Pall.) Fisch. \& C.A.Mey. & $\mathrm{H}$ & & $2900-3300$ & $1,2,3,6,7,13$ & $\begin{array}{l}\text { SU20190410, } \\
\text { SU20201206 }\end{array}$ \\
\hline Rhodiola rosea $\mathrm{L}$. & $\mathrm{H}$ & VU & $2500-3300$ & $1-8,13,14$ & SU20140412 \\
\hline Rhodiola stephani (Cham.) Trautv. \& C.A.Mey. & $\mathrm{H}$ & $\mathrm{AE}$ & $3000-3300$ & 7 & $\begin{array}{l}\text { SU20190321, } \\
\text { SU20140604 }\end{array}$ \\
\hline \multicolumn{6}{|l|}{ Cyperaceae } \\
\hline Carex atrofusca Schkuhr & $\mathrm{H}$ & & $2600-3000$ & $1,3,7$ & SU20190316 \\
\hline Carex coriophora Fisch. \& C.A.Mey. ex Kunth & $\mathrm{H}$ & & $2600-3000$ & $1,2,3,4,5,7+, 8,9$ & SU20200408 \\
\hline Carex duriuscula C.A.Mey. & $\mathrm{H}$ & & $1500-3300$ & $1-14,16$ & SU20200414 \\
\hline Carex melanocephala Turcz. & $\mathrm{H}$ & & $2600-3000$ & $1,3,7$ & $\begin{array}{l}\text { SU20201101, } \\
\text { SU20201206 }\end{array}$ \\
\hline Carex myosuroides Vill. & $\mathrm{H}$ & & $2600-3300$ & $1,2,3,5,6,7,10,13,14$ & $\begin{array}{l}\text { SU20201102, } \\
\text { SU20201211 }\end{array}$ \\
\hline Carex pediformis C.A.Mey. & $\mathrm{H}$ & & $2100-3000$ & $1-9,13,14$ & $\begin{array}{l}\text { SU20190322, } \\
\text { SU20200412 }\end{array}$ \\
\hline Eriophorum angustifolium Honck. & $\mathrm{H}$ & & $2900-3300$ & $1-7,9,10,11$ & SU20140413 \\
\hline Eriophorum bumile Turcz. & $\mathrm{H}$ & & $3000-3300$ & $1,2,3,6,7$ & SU20140414 \\
\hline
\end{tabular}


Appendix 1. Continued

\begin{tabular}{|c|c|c|c|c|c|}
\hline Taxon & $\begin{array}{l}\text { Growth } \\
\text { form }\end{array}$ & $\begin{array}{l}\text { IUCN } \\
\text { status }\end{array}$ & $\begin{array}{l}\text { Elevation, } \\
\text { m a.s.1 } \\
\text { (low to high) }\end{array}$ & Region & $\begin{array}{l}\text { Herbarium } \\
\text { code }\end{array}$ \\
\hline \multicolumn{6}{|l|}{ Euphorbiaceae } \\
\hline Euphorbia mongolica (Prokh.) Prokh. & $\mathrm{H}$ & & $1800-2500$ & $3,6,7,10,11,12,13$ & SU20190114 \\
\hline Euphorbia potaninii Prokh. & $\mathrm{H}$ & & $2600-3000$ & $3,6,7,10,13$ & SU20140313 \\
\hline \multicolumn{6}{|l|}{ Fabaceae } \\
\hline Astragalus brevifolius Ledeb. & $\mathrm{H}$ & & $1500-2500$ & $1,2,3,4,6,7+, 8,11,12,13$ & SU20200211 \\
\hline Astragalus dilutus Bunge & $\mathrm{H}$ & & $2000-2500$ & $3,6,7,10,12,13,14$ & SU20190115 \\
\hline Astragalus ellipsoideus Ledeb. & $\mathrm{H}$ & & $1500-2500$ & 7,15 & SU20190217 \\
\hline Astragalus gubanovii N.Ulziykh. & $\mathrm{H}$ & & $1500-2500$ & 7,10 & SU20140111 \\
\hline Astragalus laxmannii Jacq. & $\mathrm{H}$ & & $2000-2500$ & $1,3,6,7,8,13$ & SU20201106 \\
\hline Astragalus leptostachys Pall. & $\mathrm{H}$ & & $1600-2000$ & $3,6,7,13$ & SU20201004 \\
\hline Astragalus tschujensis Bunge & $\mathrm{H}$ & $\mathrm{AE}$ & $1800-2300$ & 7 & SU20200904 \\
\hline Astragalus vallestris Kamelin & $\mathrm{H}$ & & $1500-2000$ & $3,7,10-14$ & SU20200707 \\
\hline Caragana bungei Ledeb. & S & & $1800-2200$ & $3,6,7,10,11,13,14,15$ & SU20200606 \\
\hline Gueldenstaedtia monophylla Fisch. & $\mathrm{H}$ & VU & $2000-2100$ & $6,7,10,12,13,16$ & SU20200705 \\
\hline Hedysarum alpinum $\mathrm{L}$. & $\mathrm{H}$ & & $2900-3200$ & $1-7,9$ & $\begin{array}{l}\text { SU20190335, } \\
\text { SU20190336 }\end{array}$ \\
\hline Hedysarum inundatum Turcz. & $\mathrm{H}$ & & $2500-2800$ & $1,2,3,6,7,10,13$ & SU20140214 \\
\hline Oxytropis acanthacea Jurtzev & $\mathrm{H}$ & $\mathrm{AE}, \mathrm{NT}$ & $1500-2500$ & 6,7 & SU20201610 \\
\hline Oxytropis aciphylla Ledeb. & SS & $\mathrm{LC}$ & $1500-1800$ & $3,6,7,10-16$ & SU20200805 \\
\hline Oxytropis altaica (Pall.) Pers. & $\mathrm{H}$ & $\mathrm{AE}$ & $2000-3000$ & 6,7 & SU20200412 \\
\hline Oxytropis ampullata (Pall.) Pers. & $\mathrm{H}$ & & $1500-2000$ & $2,3,7,8,9,12,13$ & SU20200708 \\
\hline Oxytropis bungei Kom. & $\mathrm{H}$ & & $1500-2500$ & $3,6,7,8,10-14$ & SU20190216 \\
\hline Oxytropis intermedia Bunge & $\mathrm{H}$ & $\mathrm{AE}$ & $2600-3000$ & $3,6,7$ & SU20200613 \\
\hline Oxytropis oligantha Bunge & $\mathrm{H}$ & & $2600-3300$ & $3,6,7,10,13$ & SU20140516 \\
\hline Oxytropis pauciflora Bunge & $\mathrm{H}$ & & $2000-3000$ & $1,6,7,13$ & SU20140515 \\
\hline Oxytropis pumila Fisch.ex DC. & $\mathrm{H}$ & & $1600-2100$ & $3,6,7,8,10,11,13$ & SU20200706 \\
\hline Oxytropis saposhnikovii Krylov & $\mathrm{H}$ & $\mathrm{AE}, \mathrm{EN}$ & $2000-3000$ & 7,10 & SU20140303 \\
\hline Oxytropis sutaica N.Ulziykh. & $\mathrm{H}$ & $\mathrm{EN} 2$ & $2500-3100$ & 3,7 & SU20140610 \\
\hline Oxytropis trichophysa Bunge & $\mathrm{H}$ & & $2000-2500$ & $3,6,7,10,11,13,14$ & SU20190117 \\
\hline Oxytropis tschujae Bunge & $\mathrm{H}$ & $\mathrm{AE}$ & $2000-2500$ & 1,7 & SU20190216 \\
\hline Trifolium eximium Stephan ex Ser. & $\mathrm{H}$ & & $3100-3300$ & $1,2,3,4,6-11,13$ & SU20140317 \\
\hline Vicia costata Ledeb. & $\mathrm{H}$ & & $1500-2800$ & $2,3,4,6-14,16$ & SU20140214 \\
\hline \multicolumn{6}{|l|}{ Gentianaceae } \\
\hline Comastoma falcatum (Turcz.) Toyok. & $\mathrm{H}$ & & $2600-3300$ & $1,6,7,13$ & SU20190335 \\
\hline Comastoma tenellum (Rottb.) Toyok. & $\mathrm{H}$ & & $2500-3000$ & $1,2,3,6,7,13$ & SU20200513 \\
\hline Gentiana algida Pall. & $\mathrm{H}$ & $\mathrm{EN}$ & $2950-3300$ & $1,2,3,6,7,13$ & SU20140703 \\
\hline Gentiana aquatica var. pseudoaquatica (Kusn.) S.Agrawal & $\mathrm{H}$ & & $2600-3000$ & $1,2,3,4,6,7,8,9,13$ & SU20201105 \\
\hline Gentiana decumbens L.f. & $\mathrm{H}$ & & $1500-2800$ & $1-11,13,14$ & SU20140104 \\
\hline Gentiana riparia Kar. \& Kir. & $\mathrm{H}$ & $\mathrm{CR}$ & $3000-3100$ & 7,14 & SU20201104 \\
\hline Gentianella amarella subsp. acuta (Michx.) J.M.Gillett & $\mathrm{H}$ & & $2100-2500$ & $1,2,3,4,6,7,8,9,13$ & SU20201403 \\
\hline Gentianella azurea (Bunge) Holub & $\mathrm{H}$ & & $2600-3100$ & $2,3,6,7,13$ & SU20200112 \\
\hline Gentianopsis barbata (Froel.) Ma & $\mathrm{H}$ & $\mathrm{LC}$ & $2100-2500$ & $1-11,13,14$ & SU20140207 \\
\hline Lomatogonium carinthiacum (Wulfen) A.Braun & $\mathrm{H}$ & & $2600-2900$ & $1,2,3,4,6,7,8,11,13$ & SU20140417 \\
\hline \multicolumn{6}{|l|}{ Geraniaceae } \\
\hline Erodium tibetanum Edgew. \& Hook.f. & $\mathrm{H}$ & & $1500-2300$ & $4,6,7,8,10-16$ & SU20140208 \\
\hline Geranium pratense $\mathrm{L}$. & $\mathrm{H}$ & & 2100-2800 & $1,2,3,4,6,7,8,9,12,13$ & SU20200110 \\
\hline Geranium pseudosibiricum J.Mayer & $\mathrm{H}$ & & $2100-2800$ & $1-8,10$ & SU20200111 \\
\hline \multicolumn{6}{|l|}{ Grossulariaceae } \\
\hline Ribes aciculare $\mathrm{Sm}$. & S & NT & $2000-2800$ & $2,3,4,6,7,8,10,13,14$ & $\begin{array}{l}\text { SU20140315, } \\
\text { SU20140316 }\end{array}$ \\
\hline Ribes petraeum Wulfen & S & & $2000-2600$ & $1,2,3,4,7,14$ & SU20140416 \\
\hline \multicolumn{6}{|l|}{ Iridaceae } \\
\hline Iris potaninii Maxim. & $\mathrm{H}$ & $\mathrm{LC}$ & $1500-2400$ & $1,2,3,4,6-13$ & SU20190115 \\
\hline \multicolumn{6}{|l|}{ Juncaceae } \\
\hline \multirow{2}{*}{\multicolumn{6}{|c|}{$\begin{array}{l}\text { Lamiaceae } \\
\text { Ling }\end{array}$}} \\
\hline & & & & & \\
\hline Dracocephalum fruticulosum Stephan ex Willd. & SS & & $1600-2400$ & $3,4,6,7,8,10-13,16$ & SU20190118 \\
\hline Dracocephalum imberbe Bunge & $\mathrm{H}$ & & $2100-2500$ & $1,6,7$ & SU20190120 \\
\hline Dracocephalum origanoides Stephan ex Wild. & $\mathrm{H}$ & & $2600-3250$ & $1,3,4,6,7,8,9,13,14$ & SU20190334 \\
\hline $\begin{array}{l}\text { Dracocephalum origanoides subsp. bungeanum (Schischk. \& Serg.) } \\
\text { A.L.Budantsev }\end{array}$ & $\mathrm{H}$ & & $2000-2500$ & $1,6,7,13$ & SU20190416 \\
\hline Lagochilus ilicifolius Bunge ex Benth. & $\mathrm{H}$ & & $1800-2100$ & $3,7,8,10-16$ & SU20190113 \\
\hline Lagopsis marrubiastrum (Stephan) Ikonn.-Gal. & $\mathrm{H}$ & & $2600-3250$ & $3,6,7,13,14$ & $\begin{array}{l}\text { SU20190330, } \\
\text { SU20200115 }\end{array}$ \\
\hline
\end{tabular}


Appendix 1. Continued

\begin{tabular}{|c|c|c|c|c|c|}
\hline Taxon & $\begin{array}{l}\text { Growth } \\
\text { form }\end{array}$ & $\begin{array}{l}\text { IUCN } \\
\text { status }\end{array}$ & $\begin{array}{l}\text { Elevation, } \\
\text { m a.s.l } \\
\text { (low to high) }\end{array}$ & Region & $\begin{array}{l}\text { Herbarium } \\
\text { code }\end{array}$ \\
\hline Nepeta sibirica L. & $\mathrm{H}$ & & $1800-2250$ & $2,3,6,7,10,13,14$ & $\begin{array}{l}\text { SU20190122, } \\
\text { SU20200806 }\end{array}$ \\
\hline Panzerina canescens (Bunge) Sojak & $\mathrm{H}$ & NT & $1600-2200$ & $6,7,10,13$ & SU20140213 \\
\hline Panzerina lanata (L.) Soják & $\mathrm{H}$ & & $1500-2150$ & $2,3,4,6-14,16$ & SU20200807 \\
\hline Ziziphora pamiroalaica Juz. & $\mathrm{H}$ & & $2150-2500$ & 7,14 & SU20200121 \\
\hline \multicolumn{6}{|l|}{ Liliaceae } \\
\hline Gagea serotina (L.) Ker Gawl. & $\mathrm{H}$ & & $2600-3100$ & $1,2,3,6,7,13$ & SU20140515 \\
\hline Tulipa uniflora (L.) Besser ex Baker & $\mathrm{H}$ & VU & $2000-2800$ & $3,5,7,8,9,10,14$ & SU20190114 \\
\hline \multicolumn{6}{|l|}{ Orchidaceae } \\
\hline Neottia camtschatea (L.) Rchb.f. & $\mathrm{H}$ & $\mathrm{CR}$ & $2100-3100$ & $1,2,3,7$ & SU20200512 \\
\hline \multicolumn{6}{|l|}{ Orobanchaceae } \\
\hline Euphrasia pectinata Ten. & $\mathrm{H}$ & & $2000-2200$ & $1-10,13,14$ & SU20200710 \\
\hline Orobanche cernua Loefl. & $\mathrm{H}$ & & $1850-2100$ & $5,7-15$ & SU20140315 \\
\hline Pedicularis abrotanifolia M.Bieb. ex Steven & $\mathrm{H}$ & LC & $2000-2500$ & $1,3,6,7,13,14$ & SU20200511 \\
\hline Pedicularis achilleifolia Stephan ex Willd. & $\mathrm{H}$ & & $2900-3250$ & $1,3,6,7,8,10,14$ & SU20140514 \\
\hline Pedicularis amoena Adams ex Steven & $\mathrm{H}$ & & $2850-3300$ & $1,2,3,6,7,13,14$ & SU20190329 \\
\hline Pedicularis flava Pall. & $\mathrm{H}$ & & $2600-3250$ & $2,3,4,6-11,13,15$ & SU20140608 \\
\hline Pedicularis moschata Maxim. & $\mathrm{H}$ & $\mathrm{AE}$ & $2150-2500$ & $6,7,10$ & SU20190328 \\
\hline Pedicularis oederi Vahl & $\mathrm{H}$ & & $3100-3250$ & $1,2,3,6,7$ & SU20140414 \\
\hline Pedicularis sibirica Vved. & $\mathrm{H}$ & & $3000-3280$ & $1,3,7$ & SU20201103 \\
\hline Pedicularis uliginosa Bunge & $\mathrm{H}$ & & $2700-3020$ & $1,2,3,4,6,7,10,13$ & SU20190327 \\
\hline \multicolumn{6}{|l|}{ Papaveraceae } \\
\hline Corydalis inconspicua Bunge ex Ledeb. & $\mathrm{H}$ & $\mathrm{VU}$ & $3000-3350$ & $1,2,7$ & SU20190413 \\
\hline Hypecoum lactiflorum (Kar. \& Kir.) Pazij & $\mathrm{H}$ & & $1600-2100$ & $3,4,6-16$ & SU20140111 \\
\hline Papaver chakassicum Peschkova & $\mathrm{H}$ & & $2980-3250$ & 6,7 & SU20140314 \\
\hline Papaver lapponicum (Tolm.) Nordh. & $\mathrm{H}$ & & $3000-3300$ & 7 & SU20190326 \\
\hline Papaver pseudocanescens Popov & $\mathrm{H}$ & & $2500-3200$ & $1-7,13$ & $\begin{array}{l}\text { SU20190325, } \\
\text { SU20201409 }\end{array}$ \\
\hline \multicolumn{6}{|l|}{ Plantaginaceae } \\
\hline Lagotis integrifolia (Wild.) Schischk. & $\mathrm{H}$ & & $2900-3150$ & $1,2,3,7,13$ & SU20140607 \\
\hline Linaria altaica Fisch. & $\mathrm{H}$ & & $2050-2500$ & $3,6,7,10,14$ & SU20140513 \\
\hline Linaria hepatica Bunge & $\mathrm{H}$ & NT & $2100-2550$ & $6,7,11,13,14$ & SU20190110 \\
\hline Plantago depressa Willd. & $\mathrm{H}$ & & $2000-2500$ & $1-10,12,13$ & SU20190109 \\
\hline Plantago komarovii Pavlov & $\mathrm{H}$ & NT & $2000-2500$ & $1,3,6,7,13$ & SU20190325 \\
\hline Plantago major $\mathrm{L}$. & $\mathrm{H}$ & & $1800-2100$ & $2-14$ & SU20200109 \\
\hline Veronica ciliata Fisch. & $\mathrm{H}$ & & $3100-3200$ & $1,2,3,6,7$ & SU20200414 \\
\hline Veronica densiffora $\mathrm{Ldb}$. & $\mathrm{H}$ & & $2000-2500$ & 2,7 & SU20140107 \\
\hline Veronica spicata $\mathrm{L}$. & $\mathrm{H}$ & VU & $2100-2550$ & 7,14 & SU20190411 \\
\hline \multicolumn{6}{|l|}{ Plumbaginaceae } \\
\hline Limonium congestum (Ledeb.) Kuntze & $\mathrm{H}$ & $\mathrm{AE}$ & $2900-3150$ & $6,7,10$ & SU20190215 \\
\hline \multicolumn{6}{|l|}{ Poaceae } \\
\hline Agropyron cristatum (L.) Gaertn. & $\mathrm{H}$ & & $1500-2800$ & $1-16$ (all regions) & $\begin{array}{l}\text { SU20190211, } \\
\text { SU20200612 }\end{array}$ \\
\hline Alopecurus magellanicus Lam. & $\mathrm{H}$ & & $2600-3250$ & $1,2,3,4,6,7,8,13$ & SU20140416 \\
\hline Anthoxanthum glabrum (Trin.) Veldkamp & $\mathrm{H}$ & & $2100-2500$ & $1-10$ & SU20200512 \\
\hline Anthoxanthum monticola (Bigelow) Veldkamp & $\mathrm{H}$ & & $2600-3200$ & $1,2,3,6,7,10$ & SU20200511 \\
\hline Anthoxanthum nipponicum Honda & $\mathrm{H}$ & & $2050-3000$ & $2-7$ & SU20201212 \\
\hline Cleistogenes songorica (Roshev.) Ohwi & $\mathrm{H}$ & & $1500-2450$ & $4,7-16$ & SU20200805 \\
\hline Elymus sibiricus L. & $\mathrm{H}$ & & $1600-2000$ & $1-10,13-16$ & SU20140208 \\
\hline Festuca altaica Trin. & $\mathrm{H}$ & & $1900-2550$ & $1,3,4,6,7$ & $\begin{array}{l}\text { SU20190214, } \\
\text { SU20201408 }\end{array}$ \\
\hline Festuca lenensis Drobow & $\mathrm{H}$ & & $1500-2200$ & $1-9,13,15$ & SU20200804 \\
\hline Hordeum brevisubulatum (Trin.) Link & $\mathrm{H}$ & & $1500-2000$ & $1-14,16$ & $\begin{array}{l}\text { SU20190213, } \\
\text { SU20200209 }\end{array}$ \\
\hline Koeleria altaica (Domin) Krylov & $\mathrm{H}$ & & $2000-3000$ & $1-10,13$ & SU20190324 \\
\hline Koeleria macrantha (Ledeb.) Schult. & $\mathrm{H}$ & & $1600-2500$ & $1,2,3,4,5,7+, 8-13$ & SU20201103 \\
\hline Leymus chinensis (Trin.) Tzvelev & $\mathrm{H}$ & & $1500-2100$ & $1-6,7+, 8-14$ & SU20201306 \\
\hline Leymus secalinus (Georgi) Tzvelev & $\mathrm{H}$ & & $1500-2000$ & $1,2,3,4,6-16$ & SU20200605 \\
\hline Neotrinia splendens (Trin.) M.Nobis, P.D.Gudkova \& A.Nowak & $\mathrm{H}$ & & $1500-2100$ & $2,3,4,5,7-16$ & SU20200704 \\
\hline Phleum alpinum $\mathrm{L}$. & $\mathrm{H}$ & VU & $2000-3000$ & 7 & SU20140607 \\
\hline Poa alpina $\mathrm{L}$. & $\mathrm{H}$ & & $2000-3000$ & $1,3,6,7,15$ & SU20200208 \\
\hline Poa attenuata Trin. & $\mathrm{H}$ & & $1500-2500$ & $1,2,3,4,6-10,12-15$ & $\begin{array}{l}\text { SU20190323, } \\
\text { SU20201201' }\end{array}$ \\
\hline Poa glauca subsp. altaica (Trin.) Olonova \& G.H.Zhu & $\mathrm{H}$ & & $1500-3000$ & $1,2,3,6,7,10,13$ & $\begin{array}{l}\text { SU20140414, } \\
\text { SU20201003 }\end{array}$ \\
\hline
\end{tabular}


Appendix 1. Continued

\begin{tabular}{|c|c|c|c|c|c|}
\hline Taxon & $\begin{array}{l}\text { Growth } \\
\text { form }\end{array}$ & $\begin{array}{l}\text { IUCN } \\
\text { status }\end{array}$ & $\begin{array}{l}\text { Elevation, } \\
\text { m a.s.1 } \\
\text { (low to high) }\end{array}$ & Region & $\begin{array}{l}\text { Herbarium } \\
\text { code }\end{array}$ \\
\hline Ptilagrostis mongholica (Turcz. ex Trin.) Griseb. & $\mathrm{H}$ & LC & $1500-2000$ & $1,2,3,4,7$ & $\begin{array}{l}\text { SU20190322, } \\
\text { SU20200611, }\end{array}$ \\
\hline Stipa glareosa P.A.Smirn. & $\mathrm{H}$ & & $1500-2100$ & $3,6-16$ & SU20200801 \\
\hline Stipa krylovii Roshev. & $\mathrm{H}$ & & $1500-2000$ & $1-14$ & SU20190212 \\
\hline Trisetum spicatum (L.) K.Richt. & $\mathrm{H}$ & & $2600-3100$ & $1,2,3,4,6,7,13$ & SU20140605 \\
\hline \multicolumn{6}{|l|}{ Polygonaceae } \\
\hline Atraphaxis pungens (M.Bieb.) Jaub. \& Spach & S & & $1800-2100$ & $2-16$ & SU20200611 \\
\hline $\begin{array}{l}\text { Bistorta elliptica (Willd. ex Spreng.) V.V.Petrovsky, D.F.Murray \& } \\
\text { Elven }\end{array}$ & $\mathrm{H}$ & & $2600-3300$ & $1,2,3,6,7$ & SU20140309 \\
\hline Bistorta vivipara (L.) Delarbre & $\mathrm{H}$ & $\mathrm{LC}$ & $2500-3250$ & $1-4,6,7,8,10,13,14$ & $\begin{array}{l}\text { SU20190320, } \\
\text { SU20201101 }\end{array}$ \\
\hline Koenigia alpina (All.) T.M.Schust. \& Reveal & $\mathrm{H}$ & & $2600-3300$ & $1,2,3,4,6,7,8,14$ & SU20190114 \\
\hline Oxyria digyna (L.) Hill & $\mathrm{H}$ & & $3000-3300$ & $1,2,3,6,7,13$ & $\begin{array}{l}\text { SU20190317, } \\
\text { SU20201205 }\end{array}$ \\
\hline Persicaria minor (Huds.) Opiz & $\mathrm{H}$ & & $2500-2800$ & $7+, 10,14$ & SU20140207 \\
\hline Rheum compactum $\mathrm{L}$. & $\mathrm{H}$ & & $2600-3150$ & $1,2,3,4,6,7,12,13,14$ & SU20190318 \\
\hline Rheum nanum Siev.ex Pall. & $\mathrm{H}$ & & $1500-2000$ & $7,8,10-16$ & SU20200701 \\
\hline Rumex thyrsiflorus Fingerh. & $\mathrm{H}$ & & $2850-3200$ & $1-14$ & SU20190319 \\
\hline \multicolumn{6}{|l|}{ Primulaceae } \\
\hline Androsace bungeana Schischk. \& Bobrov & $\mathrm{H}$ & & $2000-2600$ & $1,2,3,6,7,9$ & SU20190319 \\
\hline Androsace dasyphylla Bunge & $\mathrm{H}$ & & $2600-3280$ & $1,2,3,6,7,13$ & SU20140207 \\
\hline Androsace lactiflora Fisch. ex Willd. & $\mathrm{H}$ & & $2300-3000$ & $1,2,3,4,6,7,9,14$ & $\begin{array}{l}\text { SU20190316, } \\
\text { SU20200208 }\end{array}$ \\
\hline Androsace lehmanniana Spreng. & $\mathrm{H}$ & & $2800-3300$ & $1,2,3,6,7,9$ & SU20201306 \\
\hline Androsace maxima $\mathrm{L}$. & $\mathrm{H}$ & & $2500-3200$ & $2,3,4,6-10,13,14,15$ & SU20140308 \\
\hline Androsace septentrionalis $\mathrm{L}$. & $\mathrm{H}$ & & $2300-3000$ & $1-9,12,13$ & SU20200605 \\
\hline Primula algida Adams & $\mathrm{H}$ & & $2900-3250$ & $3,6,7$ & SU20140513 \\
\hline Primula bukukunica Kovt. & $\mathrm{H}$ & & $3000-3280$ & $7,11,13$ & SU20190410 \\
\hline Primula longiscapa Ledeb. & $\mathrm{H}$ & & $2100-2800$ & $3,6,7,10,13,14$ & SU20200319 \\
\hline Primula nivalis Pall. & $\mathrm{H}$ & & $3050-3220$ & $1,2,3,6,7,10$ & SU20140512 \\
\hline \multicolumn{6}{|l|}{ Ranunculaceae } \\
\hline Aconitum glandulosum Rapaics & $\mathrm{H}$ & & $2600-3000$ & $1,2,3,4,6,7,13,14$ & SU20140508 \\
\hline Clematis alpina subsp. sibirica (L.) Kuntze & SS & & $2100-2500$ & $1,2,3,4,6,7,8,10,13$ & SU20140301 \\
\hline Delphinium crassifolium Schrad. ex Spreng. & $\mathrm{H}$ & & $2800-3200$ & $1,2,3,5,6,7$ & SU20140407 \\
\hline Delphinium inconspicuum Serg. & $\mathrm{H}$ & $\mathrm{AE}$ & $2800-3100$ & $3,6,7,14$ & SU20140408 \\
\hline Halerpestes sarmentosa (Adams) Kom. & $\mathrm{H}$ & & $2600-3280$ & $3,4,6-16$ & SU20201305 \\
\hline Leptopyrum fumarioides (L.) Rchb. & $\mathrm{H}$ & & $1600-2200$ & $1,2,3,4,6,7,8,9,13$ & SU20200901 \\
\hline Pulsatilla bungeana C.A.Mey. & $\mathrm{H}$ & & $1800-2100$ & $4,6,7$ & SU20140205 \\
\hline Ranunculus longicaulis Ledeb. ex A.Spreng. & $\mathrm{H}$ & & $2300-2800$ & $1,2,3,6,7,11,14$ & SU20140509 \\
\hline Ranunculus pedatifidus $\mathrm{Sm}$. & $\mathrm{H}$ & & $2400-2900$ & $1-7,9,13$ & SU20200407 \\
\hline Ranunculus pseudobirculus Schrenk. & $\mathrm{H}$ & & $3100-3300$ & $1,2,3,6,7,13,14$ & SU20140411 \\
\hline Ranunculus sapozhnikovii Schegol. & $\mathrm{H}$ & $\mathrm{AE}$ & $3080-3250$ & 7 & SU20140409 \\
\hline Thalictrum alpinum $\mathrm{L}$. & $\mathrm{H}$ & & $2500-3280$ & $1,2,3,4,6,7,13$ & $\begin{array}{l}\text { SU20190303, } \\
\text { SU20201207 }\end{array}$ \\
\hline Thalictrum foetidum $\mathrm{L}$. & $\mathrm{H}$ & & $2100-2800$ & $1,2,3,4,6-10,13,14$ & SU20190112 \\
\hline Trollius altaicus C.A.Mey. & $\mathrm{H}$ & & $2600-3000$ & $6,7,14$ & SU20140506 \\
\hline \multicolumn{6}{|l|}{ Rosaceae } \\
\hline Argentina anserina (L.) Rydb. & $\mathrm{H}$ & & $1600-3000$ & $1-11,13,14,15$ & SU20201303 \\
\hline Chamaerhodos erecta (L.) Bunge. & $\mathrm{H}$ & & $2100-2500$ & $1-13$ & SU20200406 \\
\hline Cotoneaster uniflorus Bunge & $\mathrm{S}$ & & $2250-2900$ & $1,2,3,7,8,13$ & SU20140510 \\
\hline Dasiphora parvifolia (Fisch. ex Lehm.) Juz. & S & & $2100-2800$ & $2,3,4,6,7+, 8$ & $\begin{array}{l}\text { SU20200409, } \\
\text { SU20200410 }\end{array}$ \\
\hline Dryas oxyodonta Juz. & SS & & $3100-3280$ & $1,2,3,4,6,7$ & SU20201208 \\
\hline Farinopsis salesoviana (Stephan) Chrtek \& Soják & SS & & $2600-3200$ & $6,7,10,13,14$ & SU20140401 \\
\hline Potentilla agrimonioides M.Bieb. & $\mathrm{H}$ & & $2500-3000$ & $6,7,14$ & SU20190111 \\
\hline Potentilla altaica Bunge & $\mathrm{H}$ & & $2000-2600$ & $1,6,7$ & SU20190110 \\
\hline Potentilla aphanes Sojak & $\mathrm{H}$ & & $2100-3000$ & $3,6,7,10,13,14$ & SU20200408 \\
\hline Potentilla astragalifolia Bunge & $\mathrm{H}$ & & $1800-2200$ & $3,6,7,10,11$ & SU20200604 \\
\hline Potentilla conferta Bunge & $\mathrm{H}$ & & $2000-3100$ & $1-9,12,13,14$ & SU20200911 \\
\hline Potentilla crebridens Juz. & $\mathrm{H}$ & & $2150-2900$ & $1,2,3,6,7$ & $\begin{array}{l}\text { SU20190210, } \\
\text { SU20200502 }\end{array}$ \\
\hline Potentilla gelida C.A.Meyer. & $\mathrm{H}$ & & $2100-2950$ & $1,2,3,6,7,9,13,14$ & $\begin{array}{l}\text { SU20140312, } \\
\text { SU20201209 }\end{array}$ \\
\hline Potentilla kryloviana Th.Wolf & $\mathrm{H}$ & & $2000-3050$ & $3,7,14$ & SU20201202 \\
\hline Potentilla nivea $\mathrm{L}$. & $\mathrm{H}$ & & $1900-3100$ & $1,2,3,4,6,7,13,14$ & $\begin{array}{l}\text { SU20190206, } \\
\text { SU20200210 }\end{array}$ \\
\hline
\end{tabular}


Appendix 1. Continued

\begin{tabular}{|c|c|c|c|c|c|}
\hline Taxon & $\begin{array}{l}\text { Growth } \\
\text { form }\end{array}$ & $\begin{array}{l}\text { IUCN } \\
\text { status }\end{array}$ & $\begin{array}{l}\text { Elevation, } \\
\text { m a.s.1 } \\
\text { (low to high) }\end{array}$ & Region & $\begin{array}{l}\text { Herbarium } \\
\text { code }\end{array}$ \\
\hline Potentilla ozjorensis Peschkova & $\mathrm{H}$ & & $2000-2950$ & $1,3,4,7$ & SU20200205 \\
\hline Potentilla pamirica Th.Wolf & $\mathrm{H}$ & & $2500-3180$ & $6,7,10,14$ & SU20201403 \\
\hline Potentilla pamiroalaica Juz. & $\mathrm{H}$ & & $2950-3200$ & 7,14 & SU20201301 \\
\hline Potentilla rhipidopbylla Sojak & $\mathrm{H}$ & $\mathrm{E}$ & $2000-2600$ & 7 & SU20190304 \\
\hline Potentilla sericea $\mathrm{L}$. & $\mathrm{H}$ & & $2100-3000$ & $1,2,3,4,6-13,15$ & SU20200405 \\
\hline Potentilla tergemina Sojak & $\mathrm{H}$ & & $2600-3100$ & $2,3,4,5,7+, 9$ & SU20190303 \\
\hline Potentilla tericholica Sobolevsk. & $\mathrm{H}$ & & $2100-2800$ & 6,7 & SU20200207 \\
\hline Potentilla turkestanica Sojak & $\mathrm{H}$ & & $2950-3200$ & 7,14 & SU20200211 \\
\hline Rosa laxa Retz. & S & NT & $2500-2800$ & $6,7,13,14,15$ & SU20140705 \\
\hline Sibbaldia adpressa (Bge.) Juz. & $\mathrm{H}$ & & $1800-2600$ & $1-13,15,16$ & SU20200606 \\
\hline Sibbaldianthe bifurca (L.) Kurtto \& T.Erikss. & SS & & $1700-2500$ & $1-14$ & SU20190209 \\
\hline Spiraea alpina Pall. & $\mathrm{S}$ & & $2200-3000$ & $1,2,3,6,7$ & SU20140511 \\
\hline \multicolumn{6}{|l|}{ Rubiaceae } \\
\hline Galium verum $\mathrm{L}$. & $\mathrm{H}$ & & $1800-2500$ & $1-10,13,14$ & SU20200108 \\
\hline \multicolumn{6}{|l|}{ Salicaceae } \\
\hline Populus laurifolia Ledeb. & $\mathrm{T}$ & & $1700-2600$ & $2,3,6,7,10,13,14$ & SU20140410 \\
\hline Salix arctica subsp. torulosa (Ledeb.) Hultén & $\mathrm{S}$ & & $3000-3250$ & $1,3,6,7$ & SU20140507 \\
\hline Salix berberifolia Pall. & S & & $3000-3100$ & $1,2,3,6,7,13$ & SU20140603 \\
\hline Salix turçaninowii Laksch. & S & & $2900-3100$ & $1,2,6,7$ & SU20140404 \\
\hline \multicolumn{6}{|l|}{ Saxifragaceae } \\
\hline Saxifraga cernua L. & $\mathrm{H}$ & & $2500-3000$ & $1,2,3,6,7,8,9,13$ & $\begin{array}{l}\text { SU20190316, } \\
\text { SU20201402 }\end{array}$ \\
\hline Saxifraga birculus L. & $\mathrm{H}$ & $\mathrm{EN}$ & $2800-3100$ & $1-7,13$ & SU20190401 \\
\hline Saxifraga macrocalyx Tolm. & $\mathrm{H}$ & & $2900-3150$ & $1,6,7,13,14$ & SU20190314 \\
\hline Saxifraga sibirica $\mathrm{L}$. & $\mathrm{H}$ & & $3000-3200$ & $1,3,6,7,10,13,14$ & $\begin{array}{l}\text { SU20190302, } \\
\text { SU20201401 }\end{array}$ \\
\hline \multicolumn{6}{|l|}{ Solanaceae } \\
\hline Physocblaina physaloides (L.) G.Don & $\mathrm{H}$ & & $1800-2200$ & $1,3-9,11,12,13$ & SU20140201 \\
\hline \multicolumn{6}{|l|}{ Ulmaceae } \\
\hline Ulmus pumila L. & $\mathrm{T}$ & & $1600-2500$ & $2-5,7,8,9,11,13,16$ & SU20140606 \\
\hline \multicolumn{6}{|l|}{ Urticaceae } \\
\hline Urtica cannabina $\mathrm{L}$. & $\mathrm{H}$ & & $1800-2600$ & $2-10,12,13,14$ & SU20200104 \\
\hline \multicolumn{6}{|l|}{ Viburnaceae } \\
\hline Adoxa moschatellina $\mathrm{L}$. & $\mathrm{H}$ & & $2200-2500$ & $1,2,3,5,6,7,13$ & SU20200506 \\
\hline \multicolumn{6}{|l|}{ Violaceae } \\
\hline Viola dissecta Ledeb. & $\mathrm{H}$ & & $2800-3100$ & $1-5,7,9,13$ & SU20140602 \\
\hline \multicolumn{6}{|l|}{ Zygophyllaceae } \\
\hline Zygophyllum melongena Bunge & $\mathrm{H}$ & & $1500-2100$ & $3,6,7,10,11,13,14$ & SU20140106 \\
\hline
\end{tabular}

